\title{
Geodesic planes in hyperbolic 3-manifolds
}

\section{Citation}

McCullen, Curtis T., Amir Mohammadi, and Hee Oh. 2015. Geodesic planes in hyperbolic 3manifolds. Working paper, Harvard University.

\section{Permanent link}

http://nrs.harvard.edu/urn-3:HUL.InstRepos:24981207

\section{Terms of Use}

This article was downloaded from Harvard University's DASH repository, and is made available under the terms and conditions applicable to Other Posted Material, as set forth at http:// nrs.harvard.edu/urn-3:HUL.InstRepos:dash.current.terms-of-use\#LAA

\section{Share Your Story}

The Harvard community has made this article openly available.

Please share how this access benefits you. Submit a story.

Accessibility 


\title{
Geodesic planes in hyperbolic 3-manifolds
}

\author{
Curtis T. McMullen, Amir Mohammadi and Hee Oh
}

28 April 2015

\section{Contents}

1 Introduction . . . . . . . . . . . . . . . . . 1

2 Background ....................... 8

3 Fuchsian groups . . . . . . . . . . . . . . 11

4 Zariski dense Kleinian groups . . . . . . . . . . . . . . 13

5 Influence of a Fuchsian subgroup . . . . . . . . . . . . . 16

6 Convex cocompact Kleinian groups . . . . . . . . . . . 18

$7 \quad$ Acylindrical manifolds . . . . . . . . . . . . . . . . . 20

8 Unipotent blowup . . . . . . . . . . . . . . . . 21

9 No exotic circles . . . . . . . . . . . . . 26

10 Thin Cantor sets . . . . . . . . . . . . . . . 31

11 Isolation and finiteness . . . . . . . . . . . . . 32

A Appendix: Quasifuchsian groups ............ 34

B Appendix: Bounded geodesic planes . . . . . . . . . 36

Research supported in part by the Alfred P. Sloan Foundation (A.M.) and the NSF. Revised 27 Nov, 2015. 


\section{Introduction}

Let $M=\Gamma \backslash \mathbb{H}^{3}$ be an oriented complete hyperbolic 3-manifold, presented as the quotient of hyperbolic space by the action of a Kleinian group

$$
\Gamma \subset G=\mathrm{PGL}_{2}(\mathbb{C}) \cong \operatorname{Isom}^{+}\left(\mathbb{H}^{3}\right) .
$$

Let

$$
f: \mathbb{H}^{2} \rightarrow M
$$

be a geodesic plane, i.e. a totally geodesic immersion of a hyperbolic plane into $M$.

We often identify a geodesic plane with its image, $P=f\left(\mathbb{H}^{2}\right)$. One can regard $P$ as a $2-$ dimensional version of a Riemannian geodesic. It is natural to ask what the possibilities are for its closure,

$$
V=\overline{f\left(\mathbb{H}^{2}\right)} \subset M
$$

When $M$ has finite volume, Shah and Ratner showed that strong rigidity properties hold: either $V=M$ or $V$ is a properly immersed surface of finite area [Sh], [Rn2]. Our goal in this paper is to show that the same type of rigidity persists for certain infinite volume hyperbolic 3-manifolds.

Planes in acylindrical manifolds. To state the main results, recall that the convex core of $M$ is given by:

$$
\operatorname{core}(M)=\Gamma \backslash \operatorname{hull}(\Lambda) \subset M,
$$

where $\Lambda \subset S^{2}$ is the limit set of $\Gamma$, and $\operatorname{hull}(\Lambda) \subset \mathbb{H}^{3}$ is its convex hull. The ends of $M$ are the open components $E$ of $M-\operatorname{core}(M)$.

Let us say $M$ is a rigid acylindrical manifold if the following two equivalent conditions hold:

1. The convex core of $M$ is a compact submanifold of $M$ with nonempty, totally geodesic boundary.

2. The Kleinian group $\Gamma$ is geometrically finite, without cusps, and its domain of discontinuity

$$
\Omega=S^{2}-\Lambda=\bigcup_{1}^{\infty} B_{i}
$$

is a countable union of round disks with disjoint closures. 
These conditions imply that $M$ is acylindrical in the sense of [Th2], and that $\Lambda$ is a Sierpiński universal curve [Why]; see Figure 1. We call such a manifold rigid because the double of its convex core is a closed hyperbolic manifold, to which Mostow rigidity applies. In particular, there are only countably many rigid acylindrical hyperbolic 3-manifolds, up to isometry. Moreover, any convex cocompact acylindrical hyperbolic manifold can be deformed to a unique rigid acylindrical manifold [Mc1, Cor 4.3].

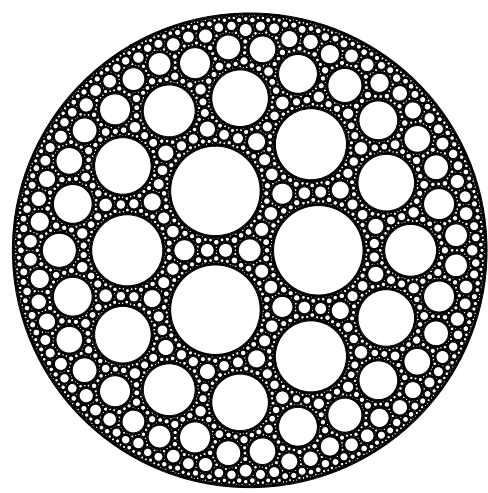

Figure 1. The limit set $\Lambda$ for a rigid acylindrical 3-manifold.

We may now state:

Theorem 1.1 Let $f: \mathbb{H}^{2} \rightarrow M$ be a geodesic plane in a rigid acylindrical hyperbolic 3-manifold, and let $V=\overline{f\left(\mathbb{H}^{2}\right)}$. Then either:

1. $V$ is an immersed compact hyperbolic surface; or

2. $V$ is a properly immersed, nonelementary, convex cocompact hyperbolic surface of infinite volume; or

3. $V=M$; or

4. $V=\bar{E}$ for some end $E$ for $M$; or

5. $V$ is a hyperbolic plane, properly immersed into one of the ends of $M$.

Corollary 1.2 The closure of a geodesic plane in $M$ is a properly immersed submanifold of $M$. 
Bounded planes. Note that $P=f\left(\mathbb{H}^{2}\right)$ is closed except in cases (3) and (4). In case (4), the closure is an embedded submanifold with boundary. We remark that the geodesic plane $P$ is bounded only in case (1), and a rather general result holds for such planes:

Theorem 1.3 Let $P \subset M=\Gamma \backslash \mathbb{H}^{3}$ be a bounded geodesic plane in an arbitrary hyperbolic 3-manifold. Then either $P$ is a compact immersed surface, or $\bar{P}=M$.

See Appendix B. In the rigid acylindrical case, the components of $\partial$ core $(M)$ give compact geodesic surfaces in $M$, but there may be others. Note also that $V$ may be non-orientable, even though $M$ is orientable.

Isolation and finiteness. The proof of Theorem 1.1 uses, in part, an isolation theorem for compact geodesic surfaces, which also leads to the following finiteness result:

Theorem 1.4 Let $M=\Gamma \backslash \mathbb{H}^{3}$ be an infinite volume hyperbolic 3-manifold with compact convex core. Then $M$ contains only finitely many compact, immersed geodesic surfaces.

In particular, case (1) of Theorem 1.1 occurs for only finitely many $V \subset M$. On the other hand, in arithmetic examples case (2) can occur for infinitely many $V$. See $\S 11$ for details.

Passage to the frame bundle. To make Theorem 1.1 more precise, we reformulate it in the language of group actions on homogeneous spaces. First we identify $\mathbb{H}^{3}$ with $G / K$, where

$$
G=\operatorname{Isom}^{+}\left(\mathbb{H}^{3}\right) \cong \mathrm{PGL}_{2}(\mathbb{C})
$$

and

$$
K \cong \operatorname{Isom}^{+}\left(S^{2}\right) \cong \mathrm{SU}(2) /( \pm I) .
$$

Then $G$ itself can be regarded as the bundle of oriented frames over $\mathbb{H}^{3}$. We also let

$$
H=\operatorname{Isom}\left(\mathbb{H}^{2}\right) \cong \operatorname{PGL}_{2}(\mathbb{R}) \subset G .
$$

Note that $H$ includes the orientation-reversing isometries of $\mathbb{H}^{2}$. We have $\mathbb{H}^{2} \cong H / K_{0}$, where $K_{0}=O_{2}(\mathbb{R})$.

In this setup, the study of geodesic planes in $M=\Gamma \backslash \mathbb{H}^{3}$ lifts to the study of $H$-orbits on the frame bundle, $F M=\Gamma \backslash G$. That is, for every geodesic 
plane $f: \mathbb{H}^{2} \rightarrow M$, we have a frame $x \in F M$ such that $f$ lifts to a map $\widetilde{f}: H \rightarrow \Gamma \backslash G$ of the form

$$
\widetilde{f}(h)=\Gamma x h,
$$

making the diagram

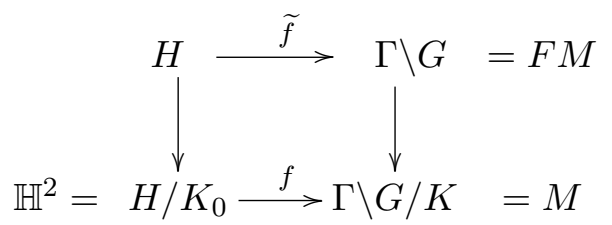

commute.

Classification of circles. Since the map $F M \rightarrow M$ is proper, the projection of the closure of $\widetilde{f}(H)$ to $M$ is the same as the closure of $f\left(\mathbb{H}^{2}\right)$. Thus the description of the closures of $H$-orbits on $\Gamma \backslash G$ refines the description of the closures of geodesic planes in $M$.

We now turn to this description. It is convenient to interchange the roles of $H$ and $\Gamma$. Then

$$
\mathcal{C}=G / H
$$

is the space of unoriented circles on $S^{2}$ (of varying radii), and we wish to describe the closures of the $\Gamma$-orbits on $\mathcal{C}$. Note that a circle $C \subset S^{2}$ bounds a unique hyperplane in $\mathbb{H}^{3}$, and hence it determines a geodesic plane

$$
f: \mathbb{H}^{2} \rightarrow M
$$

which is unique up to composition with an isometry of the domain. The map $f$ factors through the surface $S=\Gamma^{C} \backslash$ hull $(C)$, where

$$
\Gamma^{C}=\{\gamma \in \Gamma: \gamma(C)=C\},
$$

and the induced map $S \rightarrow M$ is generically one-to-one.

The five cases listed in Theorem 1.1 are then made more precise by the five cases of the following result, proved in $\S 7$.

Theorem 1.5 Let $M=\Gamma \backslash \mathbb{H}^{3}$ be a rigid acylindrical manifold, and let $\Lambda$ be the limit set of $\Gamma$. Let $C$ be a circle on $S^{2}$. Then either:

1. $C \cap \Lambda=C, \Gamma^{C}$ is conjugate to a cocompact subgroup of $\mathrm{PGL}_{2}(\mathbb{R})$, and $\Gamma C \subset \mathcal{C}$ is discrete; or

2. $C \cap \Lambda$ is a Cantor set, $\Gamma^{C}$ is conjugate to a convex cocompact subgroup of $\mathrm{PGL}_{2}(\mathbb{R})$, and $\Gamma C$ is discrete; or 
3. $C \cap \Lambda$ is a Cantor set, and

$$
\overline{\Gamma C}=\{D \in \mathcal{C}: D \cap \Lambda \neq \emptyset\} ; \quad \text { or }
$$

4. $|C \cap \Lambda|=1, C$ is tangent to the boundary of some component $B$ of $\Omega$, and

$$
\overline{\Gamma C}=\{D \in \mathcal{C}: D \cap \Lambda \neq \emptyset \text { and } D \subset \gamma \bar{B} \text { for some } \gamma \in \Gamma\} ; \quad \text { or }
$$

5. $C \cap \Lambda=\emptyset$, and $\Gamma C$ is discrete in $\mathcal{C}$.

Cantor sets. Here a Cantor set means a compact, perfect, totally disconnected set. Note that cases (2) and (3) cannot be distinguished by the topology of $C \cap \Lambda$. They can, however, be distinguished by its geometry; in $\S 10$ we will show:

Theorem 1.6 Suppose $E=C \cap \Lambda$ is a Cantor set. Then we are in case (2) if $E$ is a thin subset of $C$, and otherwise in case (3).

Here a subset $E$ of a bounded metric space $X$ is thin if there is a $k>0$ such that for all $x \in X$ and $0<r<\operatorname{diam}(X)$, there exists a $y \in X$ such that

$$
B(x, r)-E \supset B(y, k r) .
$$

Homogeneous dynamics. Let $A$ and $N$ denote the real diagonal and unipotent upper triangular subgroups of $G$, respectively $(\S 2)$. In the language of homogeneous dynamics, Theorem 1.5 implies the following result.

Corollary 1.7 Let $M=\Gamma \backslash \mathbb{H}^{3}$ be a rigid acylindrical manifold, and let

$$
X=\{x \in \Gamma \backslash G: \overline{x A} \text { is compact }\} .
$$

Then for any $x \in X$, either $x H$ is closed or $\overline{x H}=X N H$.

(Theorem 1.5 includes a description of $\overline{x H}$ in the case $x \notin X$ as well.)

The locus $X$ can be identified with the renormalized frame bundle of $M$, corresponding to geodesics with both endpoints in the limit set of $\Gamma$ (see $\S 2)$. It is also known that $\overline{x N}=X N$ for all $x \in X$; see [Fer], [Win].

Counterexamples. What happens if we drop the assumption that $M$ is a rigid acylindrical manifold? 
It is easy to see that, when $\Gamma$ is a Fuchsian surface group, there is no analogue of Theorem 1.1; the closure of a plane in such a hyperbolic 3manifold can be as badly behaved as the closure of a geodesic on a hyperbolic surface.

More precisely, let $M=\Gamma \backslash \mathbb{H}^{3}$ where $\Gamma \subset \mathrm{PSL}_{2}(\mathbb{R})$ is a cocompact Fuchsian group. Then the convex core $\Sigma \subset M$ is a compact, totally geodesic surface. Given any geodesic $\gamma \subset \Sigma$, there is a unique hyperbolic plane $P \subset M$, normal to $\Sigma$, such that $P \cap \Sigma=\gamma$. We then have

$$
\bar{P} \cong \bar{\gamma} \times \mathbb{R} \subset M
$$

Since $\bar{\gamma}$ can be a fairly wild set (e.g. locally a Cantor set times $[0,1]$ ), the closure of a geodesic plane in a Fuchsian manifold certainly need not be a submanifold, in contrast to Corollary 1.2.

This type of example is robust, in the sense that it persists for quasifuchsian groups (see Appendix A). More generally, the presence of cylinders in $M$ (in the case of incompressible boundary) seems likely to invalidate any simple description of the closures of geodesic planes in $M$ and of $H$-orbits in $\Gamma \backslash G$. Thus the requirement that $M$ is acylindrical in Theorems 1.1 and 1.5 is somewhat natural.

Discussion of the proof. To give the proof of Theorem 1.5, we first develop some general results on Kleinian groups which may be of independent interest.

Let $\Gamma \subset \mathrm{PGL}_{2}(\mathbb{C})$ be a Kleinian group with limit set $\Lambda$ and with quotient 3-manifold $M=\Gamma \backslash \mathbb{H}^{3}$. Let $C \subset S^{2}$ be a circle.

1. In $\S 3$ and $\S 4$ we describe some general mechanisms to insure that $\overline{\Gamma C} \subset \mathcal{C}$ is fairly large.

In $\S 3$ we study the case where $\Gamma$ is a (nonelementary) convex cocompact Fuchsian group, with limit set contained in a circle $D$. We observe that if $D$ is an accumulation point of $\Gamma C$, then $\overline{\Gamma C}$ contains a pencil of horocycles sweeping out at least one component of $S^{2}-D$. This statement is a direct corollary of results of Hedlund and Dal'bo.

2. Let $\mathcal{C}_{\Lambda}$ denote the set of all circles meeting the limit set $\Lambda$. In $\S 4$ we show if $\Gamma$ is Zariski dense in $\mathrm{PGL}_{2}(\mathbb{C}$ ) (as a real Lie group), and $\overline{\Gamma C}$ contains a family of circles sweeping out an open subset of $\Lambda$, then $\overline{\Gamma C} \supset \mathcal{C}_{\Lambda}$. The proof is based on the existence of a dense orbit for the geodesic flow on the renormalized frame bundle RFM. (The geodesics in the renormalized frame bundle are those which stay in the convex core of $M$ for all time; see $\S 2$.) 
3. In $\S 6$ we show that if $\Gamma$ is convex cocompact, then either $C$ is contained in $\Lambda$ or $C \cap \Lambda$ is totally disconnected. This result is needed to show that $C \cap \Lambda$ is a Cantor set in cases (2) and (3) of Theorem 1.5. The proof is based on an analysis metric density points, and shows in addition that this Cantor set has measure zero.

4. In $\S 7$ we finally turn to the case of a rigid acylindrical 3-manifold $M$. In this case the geodesic faces of $\partial \operatorname{core}(M)$ provide $\Gamma$ with many cocompact Fuchsian subgroups. Using the influence of these groups and the results above, we complete the proof of Theorem 1.5 under the assumption that $\Gamma$ has no exotic circles. Here $C$ is exotic if $\overline{\Gamma C}$ contains no discrete $\Gamma$-orbit.

5. Let $U=\left\{\left(\begin{array}{ll}1 & x \\ 0 & 1\end{array}\right): x \in \mathbb{R}\right\} \subset G$. To prepare for the proof that $\Gamma$ has no exotic circles, in $\S 8$ we prove two useful results about sequences $g_{n} \rightarrow$ id in $G$. We show that for most such sequences, one can find unipotent elements $u_{n}, u_{n}^{\prime} \in U$ such that $u_{n} g_{n} u_{n}^{\prime}$ converges to a desirable limit. These results are closely related to [Mg1, Lemma 5].

6. In $\S 9$ we complete the proof that $\Gamma$ has no exotic circles.

One of the principal challenges to understanding dynamics on an open hyperbolic 3-manifold is that the renormalized frame bundle, while invariant under the geodesic flow, is generally not invariant under the horocycle flow. We begin by showing that in the rigid acylindrical case, the horocycle flow still has good recurrence properties for RFM. The proof of recurrence uses the fact that $M$ is a rigid acylindrical manifold in two critical ways: to insure that there is a definite gap between any two distinct components of the domain of discontinuity $\Omega=S^{2}-\Lambda$; and to insure that the components of $\Omega$ are convex.

The remainder of the argument is similar to that used by Margulis in the proof of Oppenheim conjecture [Mg1]. We combine recurrence with the results of $\S 8$ to show that if $C$ is an exotic circle, then $\overline{\Gamma C}$ contains a continuous family of circles of the form $L C$, for some semigroup $L \subset G$, and that $L C$ sweeps out an open subset of $\Lambda$. But then $\overline{\Gamma C}$ contains $\mathcal{C}_{\Lambda}$ by the results of $\S 4$. In particular, we have $\partial B \in \overline{\Gamma C}$ for any component $B$ of $\Omega$. But $\Gamma \cdot \partial B$ is discrete in the space of circles, contrary to our assumption that $C$ is exotic. This completes the proof of Theorem 1.5.

Question. What can one say, in general, about the stabilizer of a circle $\Gamma^{C} \subset \Gamma$ ? For example, can $\Gamma^{C}$ be an infinitely generated group? (It is 
known that infinitely generated groups can arise in an analogous situation for Teichmüller space; cf. [Mc3]).

Notes and references. The work of Shah and Ratner establishes, more generally, the rigidity of totally geodesic $k$-manifolds in finite-volume hyperbolic $n$-manifolds, for $k \geq 2$ [Sh], [Rn2]. Payne gives a generalization to locally symmetric spaces of finite volume, using Ratner's theorems [Pa]. For an example of a compact hyperbolic 3-manifold containing no immersed compact geodesic surfaces, see [MR1, Cor. 6]. For more on homogeneous dynamics, see e.g. [Mg2], [Rn3] and [Ein]. We have chosen for directness to focus on convex cocompact groups in dimension three; many arguments in the present paper can be generalized to higher dimensions and to groups with parabolics.

In a sequel to this work [MMO], we apply the results of this paper to obtain a description of the closures of horocycles and $U$-orbits for a rigid acylindrical 3-manifold.

We would like to thank Ian Agol for raising the question of the measure of $E$ in Theorem 6.1.

\section{Background}

In this section we fix notation and recall some background in Lie groups, Kleinian groups and hyperbolic geometry. For more details see e.g. [MT], [Rc].

Lie groups. Let $G$ denote the simple, connected Lie group $\mathrm{PGL}_{2}(\mathbb{C})$. The group $G$ acts continuously on $S^{2} \cup \mathbb{H}^{3} \cong B^{3}$, preserving the hyperbolic metric on $\mathbb{H}^{3}$ and the complex structure on $S^{2} \cong \widehat{\mathbb{C}}=\mathbb{C} \cup\{\infty\}$. Indeed, as a real Lie group we have

$$
G \cong \operatorname{Aut}\left(S^{2}\right) \cong \operatorname{Isom}^{+}\left(\mathbb{H}^{3}\right) .
$$

Thus $G$ also acts on the bundle of oriented, orthonormal frames $\mathrm{FH}^{3}$ over $\mathbb{H}^{3}$. 
Within $G$, we have the following subgroups:

$$
\begin{aligned}
H & =\mathrm{PGL}_{2}(\mathbb{R}), \\
A & =\left\{\left(\begin{array}{cc}
a & 0 \\
0 & a^{-1}
\end{array}\right): a \in \mathbb{R}_{+}\right\}, \\
S^{1} & =\left\{\left(\begin{array}{cc}
\exp (i \theta) & 0 \\
0 & \exp (-i \theta)
\end{array}\right): \theta \in \mathbb{R}\right\}, \\
K & =\mathrm{SU}(2) /( \pm I), \\
N & =\left\{n_{s}=\left(\begin{array}{ll}
1 & s \\
0 & 1
\end{array}\right): s \in \mathbb{C}\right\}, \\
U & =\left\{n_{s}: s \in \mathbb{R}\right\}, \text { and } \\
V & =\left\{n_{s}: s \in i \mathbb{R}\right\} .
\end{aligned}
$$

It is understood that the matrices on the right are representatives for equivalence classes in $\mathrm{PGL}_{2}(\mathbb{C}) \cong \mathrm{SL}_{2}(\mathbb{C}) /( \pm I)$.

Homogeneous spaces. The conformal action of $G$ on $S^{2} \cong G / S^{1} A N$ extends naturally to an action by isometries on $\mathbb{H}^{3} \cong G / K$. The induced action on the frame bundle of $\mathbb{H}^{3}$ is simply transitive, and thus we can identify $G$ with $\mathrm{FH}^{3}$.

Kleinian groups and 3-manifolds. A Kleinian group is a discrete subgroup $\Gamma \subset G$. Associated to $\Gamma$ is a hyperbolic orbifold, $M=\Gamma \backslash \mathbb{H}^{3}$, with frame bundle

$$
\mathrm{F} M \cong \Gamma \backslash G .
$$

When $\Gamma$ is torsion-free, $M$ is a manifold.

The right actions of $A$ and $N$ on $\Gamma \backslash G$ give the geodesic and horocycle flows on the frame bundle, respectively. The orbit of $A$ through a frame $x=\left(e_{1}, e_{2}, e_{3}\right)$ corresponds to an oriented geodesic in the direction $e_{1}$. The orbit of $N$ through $x$ consists of those frames $y$ such that $d(x a, y a) \rightarrow 0$ as $a \rightarrow+\infty$ in $A$, where $d$ is a left invariant metric on $G$. The frame $x$ determines a unique geodesic plane $P \leftrightarrow M$ whose tangent space at one point is spanned by $\left(e_{1}, e_{2}\right)$; and $x H$ is the set of all frames tangent to $P$.

The space of circles. As in the Introduction, we let $\mathcal{C} \cong G / H$ denote the space of unoriented circles on $S^{2}$. We frequently use the following elementary fact:

If the orbit of a circle $\Gamma C \subset \mathcal{C}$ is closed, then it is discrete. 
(If $\Gamma C$ is closed but not discrete, then it has no isolated points by homogeneity, so it contains a perfect set, contrary to countability of $\Gamma$.)

The convex core. The limit set of $\Gamma$ is defined by

$$
\Lambda=S^{2} \cap \overline{\Gamma x}
$$

where $x$ is any point in $\mathbb{H}^{3}$. Here the closure is taken in the compactified hyperbolic space $S^{2} \cup \mathbb{H}^{3} \cong B^{3}$. Its complement is the domain of discontinuity,

$$
\Omega=S^{2}-\Lambda
$$

We will generally assume that $\Gamma$ is nonelementary, i.e. it does not contain an abelian subgroup of finite index. Equivalently, we assume $|\Lambda|>2$. Then:

- $\Lambda$ is a perfect set (it has no isolated points),

- The action of $\Gamma$ on $\Lambda$ is minimal (every orbit is dense), and

- The action of $\Gamma$ on $\Lambda \times \Lambda$ is transitive (there exists a dense orbit).

See e.g. [Eb, §2].

Given $E \subset S^{2}$ we let

$$
\operatorname{hull}(E) \subset \mathbb{H}^{3}
$$

denote the smallest convex set containing all geodesics with both endpoints in $E$. The convex core of $M$ is given by

$$
\operatorname{core}(M)=\Gamma \backslash \operatorname{hull}(\Lambda) \subset M \text {. }
$$

It contains all the closed geodesics in $M$.

The renormalized geodesic flow. Let $\mathrm{RF} M \subset \mathrm{F} M$ denote the renormalized frame bundle $M$, i.e. the closed set consisting of all frames $\left(e_{1}, e_{2}, e_{3}\right)$ such that $e_{1}$ is tangent to a complete geodesic $\gamma \rightarrow \operatorname{core}(M)$. The $A$-action on RFM gives the renormalized geodesic flow; cf. [Sul].

When $\Lambda \neq S^{2}$, most geodesics (a dense open set) in $M$ exit the convex core and tend to infinity in forward and backward time. The renormalized frame bundle, $\operatorname{RF} M$, captures the more recurrent part of the dynamics. It can also be described as the quotient

$$
\operatorname{RF} M=\Gamma \backslash\left\{\left(e_{1}, e_{2}, e_{3}\right) \in \mathrm{FH}^{3}: \begin{array}{l}
\text { the geodesic through } e_{1} \\
\text { has both endpoints in } \Lambda
\end{array}\right\} .
$$


The space RFM is invariant under $S^{1} \subset G$, yielding as quotient the renormalized unit tangent bundle,

$$
\mathrm{RT}_{1} M=\mathrm{RF} M / S^{1} .
$$

The fact that $\Gamma$ has a dense orbit on $\Lambda \times \Lambda$ is equivalent to the fact that there is a dense $A$-orbit in $\mathrm{RT}_{1} M$.

In general $K \subset G$ does not preserve $\operatorname{RF} M$, but we still have a natural projection

$$
\mathrm{RF} M \rightarrow \operatorname{core}(M) .
$$

This map need not be surjective.

\section{$3 \quad$ Fuchsian groups}

In this section we discuss families of circles invariant under the action of a Fuchsian group $\Gamma$.

Automorphisms of a ball. Let $B \subset S^{2}$ be an open round ball. Then $B$ carries a natural hyperbolic metric $\rho_{B}$, since it is conformally isomorphic to the upper half plane. Every isometry of $B$ extends to a conformal automorphism of $S^{2}$, and thus we have

$$
G^{B} \cong \operatorname{Isom}^{+}\left(B, \rho_{B}\right) \cong \mathrm{PSL}_{2}(\mathbb{R}) .
$$

We refer to a discrete subgroup $\Gamma \subset G^{B}$ as a Fuchsian group, since it is conjugate into $\mathrm{PSL}_{2}(\mathbb{R}) \cong \operatorname{Isom}^{+}\left(\mathbb{H}^{2}\right)$. The quotient space $X=\Gamma \backslash B$ is a hyperbolic orbifold in the metric it inherits from $B$. We usually assume $\Gamma$ is nonelementary; then its limit set $\Lambda \subset \partial B$ is either a Cantor set or the whole circle, and in particular $\Gamma$ determines the circle $\partial B$ uniquely.

Let hull $(\Lambda, B) \subset B$ denote the convex hull of the limit set in the hyperbolic metric on $B$. If $\Gamma \backslash \operatorname{hull}(\Lambda, B)$ is compact, we say $\Gamma$ is convex cocompact.

Horocycles. Now let $C$ be a circle that meets $B$. Then the intersection $C \cap B$ is an arc or circle of constant geodesic curvature in the hyperbolic metric on $B$. We say $C$ is a horocycle in $B$ if $C$ is tangent to $\partial B$; equivalently, if $C \cap B$ has geodesic curvature $k=1$.

The closure of the set of horocycles in $B$ is given by

$$
\mathcal{H}(B)=\{C \in \mathcal{C}: C \subset \bar{B} \text { and } C \cap \partial B \neq \emptyset\} .
$$

The only circle in $\mathcal{H}(B)$ which is not a horocycle, is $\partial B$ itself. Given $E \subset \partial B$, we denote the closure of the set of horocycles resting on $E$ by

$$
\mathcal{H}(B, E)=\{C \in \mathcal{H}(B): C \cap E \neq \emptyset\} .
$$



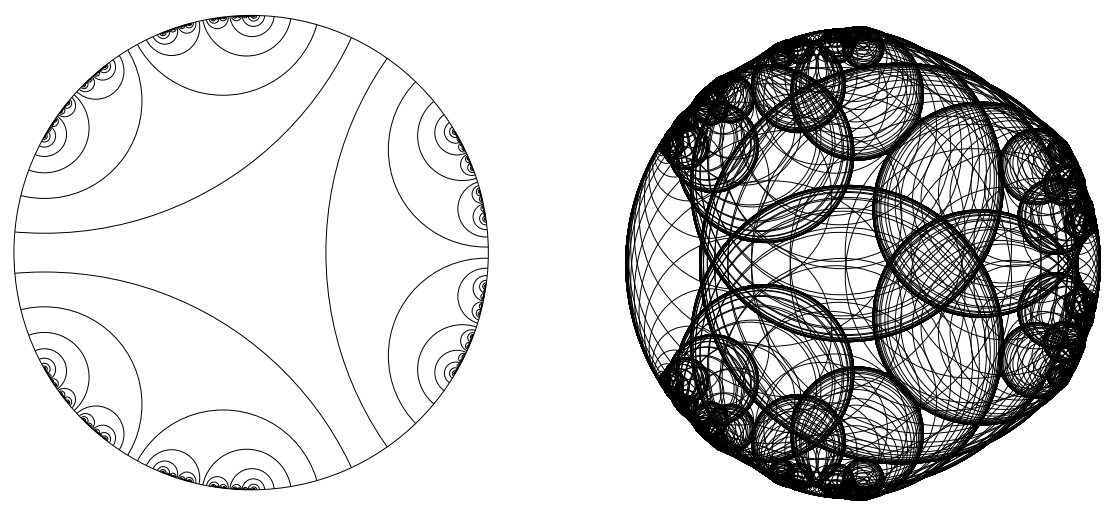

Figure 2. Dynamics of a Schottky group acting on the unit disk: orbit of a fundamental domain (left) and orbit of a circle $C$ close to $S^{1}$ (right).

Dynamics of the horocycle flow. As was shown by Hedlund, if $X=\Gamma \backslash B$ is compact, then the horocycle flow on $\mathrm{T}_{1} X$ is minimal [Hed, Thm. 2.5]. A generalization to convex cocompact groups follows from [Da, Prop. B]. Formulated in terms of the universal cover, it states the following.

Theorem 3.1 Let $\Gamma \subset G^{B}$ be a nonelementary, convex cocompact Fuchsian group, with limit set $\Lambda$. Then every $\Gamma$-orbit in the space of horocycles $\mathcal{H}(B, \Lambda)$ is dense.

Corollary 3.2 Under the same assumptions, suppose $C_{n} \rightarrow \partial B$ in $\mathcal{C}$, and $C_{n} \cap \operatorname{hull}(\Lambda, B) \neq \emptyset$ for all $n$. Then $\overline{\bigcup \Gamma C_{n}}$ contains $\mathcal{H}(B, \Lambda)$.

Proof. Let $k_{n}$ denote the geodesic curvature of $C_{n} \cap B$ in the hyperbolic metric on $B$. Since $C_{n} \rightarrow \partial B, k_{n}$ tends to 1 , the curvature of a horocycle. By assumption, $C_{n}$ also meets $\operatorname{hull}(\Lambda, B)$. Since $\Gamma$ is convex cocompact, there is a compact set $K \subset \operatorname{hull}(\Lambda, B)$ and a sequence $\gamma_{n} \in \Gamma$ such that $\gamma_{n} C_{n}$ meets $K$ for all $n$.

Choose $x \in K$ and pass to a subsequence such that $\gamma_{n} C_{n} \rightarrow D \in \mathcal{C}$ and $\gamma_{n} x \rightarrow p \in \bar{B}$. Since the hyperbolic distance $d_{B}\left(K, C_{n}\right) \rightarrow \infty$, we have $p \in \partial B \cap \overline{\Gamma x}=\Lambda$. Since $k_{n} \rightarrow 1, D$ is a horocycle for $B$; and since $C_{n}$ encloses $x$ for all $n \gg 0$, we have $p \in D$. Thus $\overline{\Gamma \bigcup C_{n}}$ contains a horocycle $D$ resting on the limit set, so it also contains all such horocycles by the preceding result.

The Corollary is illustrated in Figure 2 for a convex cocompact group acting on the unit disk. 


\section{Zariski dense Kleinian groups}

We now return to the setting of a Kleinian group $\Gamma \subset G=\mathrm{PGL}_{2}(\mathbb{C})$ with limit set $\Lambda$. Let

$$
\mathcal{C}_{\Lambda}=\{C \in \mathcal{C}: C \cap \Lambda \neq \emptyset\} .
$$

Our goal is to formulate conditions which insure that a $\Gamma$-orbit in $\mathcal{C}_{\Lambda}$ is dense.

Recall that $\Gamma$ is Zariski dense in $G=\mathrm{PGL}_{2}(\mathbb{C})$, as a real Lie group, if it is contained in no proper real algebraic subvariety $V \subset G$. It suffices to consider only subvarieties which are groups. In the case at hand, it is easy to see that $\Gamma$ is Zariski dense if and only if its limit set is not contained in a circle.

In this section we will show:

Theorem 4.1 For any Zariski dense Kleinian group $\Gamma$, there exists a dense subset $\Lambda_{0}$ of its limit set $\Lambda$ such that

$$
\overline{\Gamma C}=\mathcal{C}_{\Lambda}
$$

whenever $C$ meets $\Lambda_{0}$.

Corollary 4.2 Let $\mathcal{D} \subset \mathcal{C}$ be a collection of circles such that $\bigcup \mathcal{D}$ contains a nonempty open subset of $\Lambda$. Then there exists a $D \in \mathcal{D}$ such that $\overline{\Gamma D}=\mathcal{C}_{\Lambda}$.

Theorem 4.1 will be deduced from:

Theorem 4.3 If $\Gamma$ is Zariski dense in $G$, then there exists a dense geodesic ray in the renormalized frame bundle $\mathrm{RF} M$.

Indeed, we can take $\Lambda_{0} \subset S^{2}$ to be the orbit under $\Gamma$ of the endpoint of such a ray, when lifted to the universal cover.

Twist. Every nonparabolic element $g \in G$ is conjugate to an element am $\in A S^{1}$. We refer to $\tau(g)=m \in S^{1}$ as the twist of $g$. When $g$ is hyperbolic, $\tau(g)$ describes how a frame is rotated under parallel transport along the closed geodesic corresponding to $g$. Let $\tau(g)=$ id if $g$ is parabolic (or the identity).

Proposition 4.4 If $\Gamma$ is Zariski dense, then the twists

$$
T=\{\tau(g): g \in \Gamma \text { is hyperbolic }\}
$$

form a dense subset of $S^{1}$. 
Proof. We may assume $\Gamma$ is finitely-generated and torsion-free, since any Zariski dense discrete group contains a subgroup of this form which is still Zariski dense. Since $\tau\left(g^{n}\right)=\tau(g)^{n}, T \subset S^{1}$ is either finite or dense. But if $T$ is finite, then $\Gamma$ is contained in the variety

$$
V=\{g \in G: g \text { is parabolic, } g=\mathrm{id} \text {, or } \tau(g) \in T\},
$$

contradicting Zariski density. Thus $T$ is dense.

Corollary 4.5 Any circle meeting $\Lambda$ in 1 point is a limit of circles meeting $\Lambda$ in 2 or more points.

Proof. For each $n>0$, let $q_{n} \in \Lambda$ be the fixed point of a hyperbolic element $\gamma_{n} \in \Gamma$ such that $\tau\left(\gamma_{n}\right) \in S^{1}$ generates a subgroup of order greater than $n$. Such an element exists because $\tau(\Gamma)$ is dense in $S^{1}$.

Now suppose $C$ meets $\Lambda$ in a single point $p$. Every $\Gamma$ orbit in the limit set is dense, so we can find $a_{n} \in \Gamma q_{n}$ such that $a_{n} \rightarrow p$. Note that the tangent cone to $\Lambda$ at $a_{n}$ is invariant under $\left\langle\tau\left(q_{n}\right)\right\rangle$, so it is nearly dense in $S^{1}$ when $n \gg 0$. Thus we can find $b_{n} \in \Lambda$ such that $b_{n} \neq a_{n}, b_{n} \rightarrow p$ and $\overline{a_{n} b_{n}}$ converges to the tangent line to $C$ at $p$. Choose $c \in C$ disjoint from $\{p\},\left\{a_{n}\right\}$ and $\left\{b_{n}\right\}$, and let $C_{n} \subset S^{2}$ denote the unique circle through the three points $\left(a_{n}, b_{n}, c\right)$. Then $\left|C_{n} \cap \Lambda\right| \geq 2$ for all $n$, and by construction $C_{n} \rightarrow C$.

Proof of Theorem 4.3. Let $A_{+}=\left\{\left(\begin{array}{cc}a & 0 \\ 0 & a^{-1}\end{array}\right): a \geq 1\right\}$ denote the positive semigroup inside $A$. Introduce a relation on $\mathrm{RF} M$ by

$$
x \leq y \Longleftrightarrow \text { there exist } x_{n} \rightarrow x \text { and } y_{n} \rightarrow y \text { such that } y_{n} \in x_{n} A_{+} .
$$

Using the fact that a broken geodesic with small bends, far apart, is very close to a geodesic, one can check that this relation is transitive. It also follows directly from the definition that this relation is closed and that $x \leq$ $y \Longrightarrow x m \leq y m$ for all $m \in S^{1}$. Since $\Gamma$ is nonelementary, it has a dense orbit on $\Lambda \times \Lambda$ ( $(2)$; equivalently, there is a dense $A$-orbit in $\mathrm{RT}_{1} M$. This implies we have a dense $A_{+}$orbit as well (cf. [Eb, Prop. 3.12]), and therefore:

$$
\text { For all } x, y \in \mathrm{RF} M \text { there is an } m \in S^{1} \text { such that } x \leq y m .
$$

Given $x \in \mathrm{RF} M$, consider the closed semigroup

$$
S(x)=\left\{m \in S^{1}: x m \leq x\right\} .
$$


Since $S^{1}$ is compact, $S(x)$ is a group; and since $S^{1}$ is abelian, $S(x)=S(x m)$ for all $m \in S^{1}$. Now given any $x, y \in \mathrm{RF} M$ there exist $m_{0}, m_{1} \in S^{1}$ such that

$$
x \leq y m_{0} \leq x m_{1},
$$

with $m_{1} \in S(x)$. This implies that

$$
x \leq y m_{0} \leq y m_{0} m \leq x m_{1} m, \quad \text { for all } m \in S\left(y m_{0}\right)=S(y),
$$

and thus $S(y)$ is contained in $S(x)$. This relation holds for all $x, y \in \mathrm{RF} M$, so $S(x)$ is independent of $x$.

Now for any hyperbolic element $\gamma \in \Gamma$, there is a $y \in \operatorname{RF} M$ tangent to the geodesic stabilized by $\gamma$, and hence $\tau(\gamma) \in S(y)=S(x)$. Therefore $\tau(\Gamma) \subset S(x)$. Since $\Gamma$ is Zariski dense, $\tau(\Gamma)$ is dense in $S^{1}$ by Proposition 4.4, and hence $S(x)=S^{1}$. Thus we have $x \leq y$ for all $x, y \in \operatorname{RF} M$ by (4.1).

To complete the proof, choose a countable dense set $\left\{x_{1}, x_{2}, \ldots\right\} \subset \mathrm{RF} M$. Let $d(x, y)$ denote the natural metric on $\mathrm{F} M$, and for $i, j>0$ let

$$
U_{i j}=\left\{x \in \operatorname{RF} M: d\left(A_{+} x, x_{i}\right)<1 / j\right\} .
$$

Since $x \leq x_{i}$ for all $x$, each open set $U_{i j}$ is dense in RFM. Thus, by the Baire category theorem, $X=\bigcap_{i, j} U_{i j}$ is nonempty; and for any $x \in X$, the orbit $A_{+} x$ gives a dense geodesic ray in $\operatorname{RF} M$.

Remark: Bruhat decomposition. Transitivity of the relation $x \leq y$ can also be verified using the Bruhat decomposition. The key point is to show that, if $a_{1}, a_{2} \in A_{+}$and $g \in G$ is small (close to the identity), then $a_{1} g a_{2}=g_{1} a_{1} a_{2} g_{2}$ with $g_{1}, g_{2}$ also small. To see this, let $N_{+}$and $N_{-}$be the unipotent subgroups that are respectively expanded and contracted by the adjoint action of $A_{+}$. Using the Bruhat decomposition, we can write $g=n_{-} a m n_{+}$for small $n_{ \pm} \in N_{ \pm}, a \in A$ and $m \in S^{1}$. We then have

$$
a_{1} g a_{2}=a_{1} n_{-} a m n_{+} a_{2}=\left(n_{-}^{\prime}\right) a_{1} a_{2}\left(a m n_{+}^{\prime}\right)=g_{1} a_{1} a_{2} g_{2}
$$

and $n_{-}^{\prime}=a_{1} n_{-} a_{1}^{-1} \in N_{-}$and $n_{+}^{\prime}=a_{2}^{-1} n_{+} a_{2} \in N_{+}$are still small, by the definition of $N_{ \pm}$.

Proof of Theorem 4.1 . Let $x \in \Lambda$ be the endpoint of a geodesic ray $\gamma$ whose projection to $M$ gives a dense subset of RFM. Suppose $x \in C$.

Let $F(C) \subset \mathrm{F} M$ denote the projection to $M$ of the frames $\left(e_{1}, e_{2}, e_{3}\right) \in$ $\mathrm{FH}^{3}$ such that $e_{3}$ is normal to hyperplane $P(C)=\operatorname{hull}(C) \subset \mathbb{H}^{3}$. Since $\gamma$ and $P(C)$ are asymptotic at $x$, we have $\operatorname{RF} M \subset \overline{F(C)}$. 
Now let $D$ be a circle meeting $\Lambda$ in 2 or more points. Then the plane $P(D)$ contains a geodesic with endpoints in the limit set, and hence $F(D)$ meets RFM. Thus there is a frame in $F(D)$ which is a limit of frames in $F(C)$. This implies there are circles $C_{n} \in \Gamma C$ such that hull $\left(C_{n}\right) \rightarrow \operatorname{hull}(D)$, and hence $C_{n} \rightarrow D$.

Thus $\overline{\Gamma C}$ contains all circles meeting $\Lambda$ in 2 or more points. By Corollary 4.5, the closure also contains $\mathcal{C}_{\Lambda}$. Since $C \in \mathcal{C}_{\Lambda}$, and the latter set is closed and $\Gamma$-invariant, equality holds.

The same reasoning applies whenever $C$ meets an element of the density set $\Lambda_{0}=\Gamma x \subset \Lambda$.

Remarks. A proof of Theorem 4.3 can also be based on ergodicity of the geodesic flow on the frame bundle for Zariski dense convex cocompact Kleinian groups, established in [Win]. (Apply ergodicity to Schottky subgroups of $\Gamma$ and pass to the limit.) This second proof generalizes to all dimensions. For a stronger version of Proposition 4.4, see [PR].

\section{Influence of a Fuchsian subgroup}

In this section we combine results from the previous two sections to analyze a geodesic plane $P \subset M=\Gamma \backslash \mathbb{H}^{3}$ whose closure contains an immersed surface with nontrivial fundamental group.

The setting. Let $\Gamma$ be a Zariski dense Kleinian group with limit set $\Lambda$. Let $C \subset S^{2}$ be a circle, and suppose $D \in \overline{\Gamma C}-\Gamma C$. We wish to show that $\overline{\Gamma C}$ is large, if the stabilizer $\Gamma^{D}$ is large. We formulate two results in this direction.

Theorem 5.1 Suppose $\Gamma^{D}$ is a nonelementary group, and there is limit set on both sides of $D$. Then $\overline{\Gamma C}=\mathcal{C}_{\Lambda}$.

Proof. By assumption $\Gamma C$ is not closed, so $C$ meets $\Lambda$. Therefore

$$
\overline{\Gamma C} \subset \mathcal{C}_{\Lambda} .
$$

To establish the reverse inclusion, write the complement of $D$ as the union of two disjoint balls, $S^{2}-D=B \cup B^{\prime}$. Then $\Gamma^{B} \subset \Gamma^{D}$ is a nonelementary Fuchsian group. Any such Fuchsian group contains a nonelementary convex cocompact group $\Gamma_{0}$. Let $\Lambda_{0} \subset D$ be the limit set of $\Gamma_{0}$. 
Choose $C_{n} \in \Gamma C$ such that $C_{n} \rightarrow D$. Since $C_{n} \neq D$, we find that $C_{n}$ meets $\operatorname{hull}\left(\Lambda_{0}, B\right) \cup \operatorname{hull}\left(\Lambda_{0}, B^{\prime}\right)$ for all $n \gg 0$. (Indeed, the set

$$
S^{2}-\left(\operatorname{hull}\left(\Lambda_{0}, B\right) \cup \operatorname{hull}\left(\Lambda_{0}, B^{\prime}\right) \cup \Lambda_{0}\right)
$$

is a union of open balls, one for each component of $D-\Lambda_{0}$, whose diameters tend to zero; so eventually, $C_{n}$ is not contained in one of these balls.) By Corollary 3.2, this implies $\overline{\Gamma_{0} C}$ contains $\mathcal{H}\left(B, \Lambda_{0}\right)$ or $\mathcal{H}\left(B^{\prime}, \Lambda_{0}\right)$.

Without loss of generality, we can assume that $\mathcal{H}\left(B, \Lambda_{0}\right) \subset \overline{\Gamma_{0} C}$. Then $\overline{\Gamma C}$ contains a family of circles sweeping out the open set $B \subset S^{2}$. Since $D$ has limit set on both sides, $B$ meets $\Lambda$, and thus $\overline{\Gamma C} \supset \mathcal{C}_{\Lambda}$ by Corollary 4.2.

Immersed planes. Geometrically, Theorem 5.1 says that if $M$ contains a geodesic plane $P$ which is not closed, and its closure contains an immersed, totally geodesic surface $S$ with nonelementary fundamental group, then the chaotic dynamics on $S$ forces the nearby plane $P$ to become densely distributed in the convex core of $M$.

Boundary surfaces. The next result deals with the case where $S$ is a compact boundary component of the convex core of $M$.

Theorem 5.2 Suppose that $D=\partial B$ is the boundary of a component $B$ of $\Omega=S^{2}-\Lambda$, and $\Gamma^{D}$ is a cocompact Fuchsian group. Then either:

1. $\overline{\Gamma C}=\mathcal{C}_{\Lambda}$, or

2. $\overline{\Gamma C}=\Gamma \mathcal{H}(B)$, and $|C \cap \Lambda|=1$.

Proof. Let $B^{\prime}=S^{2}-\bar{B}$. Since $\Gamma$ is Zariski dense, its limit set is not contained in $D$, and hence $B^{\prime}$ meets $\Lambda$. Moreover $D$ is the limit set of $\Gamma^{D}$, since $\Gamma^{D}$ is cocompact.

Choose $C_{n}=\gamma_{n} C \in \Gamma C$ such that $C_{n} \rightarrow D, C_{n} \neq D$. If $C_{n}$ meets $B^{\prime}$ for infinitely many $n$, then we may repeat the previous argument: we have $\mathcal{H}\left(B^{\prime}\right) \subset \overline{\Gamma C}$ by Corollary 3.2 , and hence $\overline{\Gamma C}=\mathcal{C}_{\Lambda}$ by Corollary 4.2 .

Otherwise, we have $C_{n}=\gamma_{n} C \subset \bar{B}$ for all $n \gg 0$. Since $C_{n} \neq D$, this implies that

$$
|C \cap \Lambda|=\left|C_{n} \cap D\right|=1
$$

for all $n$, and that $C \in \Gamma \mathcal{H}(B)$. Since the diameters of the components of $\Gamma B$ tend to zero, the set $\Gamma \mathcal{H}(B)$ is closed, and hence

$$
\overline{\Gamma C} \subset \Gamma \mathcal{H}(B) .
$$


On the other hand, by Corollary 3.2 we have $\mathcal{H}(B) \subset \overline{\Gamma^{D} \bigcup C_{n}} \subset \overline{\Gamma C}$, so equality holds in the equation above.

\section{Convex cocompact Kleinian groups}

In this section we turn to the theory of convex cocompact groups, i.e. those such that the convex core of $\Gamma \backslash \mathbb{H}^{3}$ is compact. We establish the following topological and geometric properties of their limit sets.

Theorem 6.1 Let $\Gamma$ be a convex cocompact Kleinian group with limit set $\Lambda$. Then for any circle $C \subset S^{2}$, either $C$ is contained in $\Lambda$ or $C \cap \Lambda$ is a totally disconnected set of linear measure zero.

(A sharper statement is given in Corollary 6.4 below.) For later use we also record:

Theorem 6.2 Under the same hypotheses, suppose $\Gamma C$ is discrete in $\mathcal{C}$. Then $\Gamma^{C}$ is finitely generated, and $C \cap \Lambda$ is the limit set of $\Gamma^{C}$.

Points of density. Let $E$ be a closed subset of a metric space $C$. We say $c$ is a metric density point of $E$ in $C$ if

$$
\lim _{x \rightarrow c} d(x, E) / d(x, c)=0 .
$$

For example, $c=0$ is a metric density point of $E=\{0\} \cup\{1 / n: n \geq 1\}$ in $C=[0,1]$. If $C \subset S^{2}$ is a circle, then $c$ is a metric density point of $E$ if and only if

$$
\lim _{s \rightarrow \infty} d(\gamma(s), \partial \operatorname{hull}(E))=\infty
$$

for every geodesic ray $\gamma:[0, \infty) \rightarrow \operatorname{hull}(C)$ converging to $c$.

The space of inscribed circles. Now fix a convex cocompact Kleinian group $\Gamma$ with limit set $\Lambda \neq S^{2}$. Let

$$
I(\Lambda)=\{C \in \mathcal{C}: C \subset \Lambda\} .
$$

In Appendix B we will show:

The set $\Gamma \backslash I(\Lambda)$ is finite, and $\Gamma^{C} \backslash \operatorname{hull}(C)$ is compact for all $C \in$ $I(\Lambda)$. 
This means that every circle contained in $\Lambda$ corresponds to one of finitely many compact, immersed geodesic surfaces $S_{i} \subset M=\Gamma \backslash \mathbb{H}^{3}$.

Theorem 6.3 Let $C \subset S^{2}$ be a circle, let $E=C \cap \Lambda$ and let $c$ be a metric density point of $E$ in $C$. Then there is a circle $D \subset \Lambda$ tangent to $C$ at $c$.

Proof. Consider the natural projection

$$
\pi: \operatorname{hull}(C) \rightarrow M
$$

Choose a lift of $\pi$ to a continuous map $p: \operatorname{hull}(C) \rightarrow \mathrm{F} M$ such that the first two vectors in the frame $\left(e_{1}, e_{2}, e_{3}\right)=p(x)$ are tangent to the plane described by $\pi$; in other words, such that $\left(e_{1}, e_{2}\right)$ form a basis for the image of $T_{x} \operatorname{hull}(C)$ under $D \pi$.

Let $\gamma: \mathbb{R} \rightarrow \operatorname{hull}(E)$ be a geodesic such that $\gamma(s) \rightarrow c$ as $s \rightarrow+\infty$. Then $p \circ \gamma$ takes values in the compact set $\operatorname{RF} M$. Let $L \subset \operatorname{RF} M$ denote the $\omega$-limit set of $p \circ \gamma$, i.e. the set of all frames of the form $y=\lim p \circ \gamma\left(s_{n}\right)$ with $s_{n} \rightarrow \infty$. Then $L$ is compact and connected.

Since $c$ is a point of metric density, equation (6.1) holds for $\gamma(s)$. Thus for any $y \in L$ we can choose $r_{n}, s_{n} \rightarrow \infty$ such that $p \circ \gamma\left(s_{n}\right) \rightarrow y$ and the 2-dimensional balls

$$
B\left(\gamma\left(s_{n}\right), r_{n}\right) \subset \operatorname{hull}(C)
$$

are contained in hull $(E)$ for all $n$. Since hull $(E) \subset \operatorname{hull}(\Lambda)$, this means that the limiting frame $y$ must be tangent to a geodesic plane entirely contained in the convex core of $M$. Thus $y$ is tangent to one of the finitely many compact, immersed geodesic surfaces $S_{1}, \ldots, S_{n} \subset M$. Since $L$ is connected, in fact all $y \in L$ are tangent to the same surface $S_{i}$. It follows that $d\left(\pi \circ \gamma(s), S_{i}\right) \rightarrow 0$ as $s \rightarrow \infty$. Lifting to the universal cover, we find there is a circle $D \subset \Lambda$, tangent to $C$ at $c$, such that $S_{i}=\Gamma^{D} \backslash \operatorname{hull}(D)$.

Corollary 6.4 If $C$ is not contained in $\Lambda$, then $E=\Lambda \cap C$ has at most countably many metric density points.

Proof. Since $\Gamma \backslash I(\Lambda)$ is finite, $I(\Lambda)$ itself is countable, and each $D \in I(\Lambda)$ has at most one point of tangency with $C$.

Proof of Theorem 6.1. Let $E=C \cap \Lambda$. By the Lebesgue density theorem, almost every point of $E$ is a point of metric density. So if $E \neq C$, then $E$ has measure zero by the preceding Corollary, and in particular $E$ is totally disconnected. 
Proof of Theorem 6.2. Let $P=\Gamma^{C} \backslash \operatorname{hull}(C)$. Since $\Gamma C$ is discrete, the preimage of $P$ in $\mathbb{H}^{3}$ is a closed, locally finite configuration of hyperplanes. Thus the immersion $f: P \rightarrow M$ is proper. Hence $P^{\prime}=f^{-1}(\operatorname{core}(M))$ is a compact convex submanifold of $P$. The convex core of $P$ is contained in $P^{\prime}$, and hence compact, so $\pi_{1}(P) \cong \Gamma^{C}$ is finitely generated. Moreover $P^{\prime}$ lies within a bounded neighborhood of core $(P)$, so $\Lambda\left(\Gamma^{C}\right)=\Lambda(\Gamma) \cap C$.

\section{Acylindrical manifolds}

In this section we begin the proof of our main result, Theorem 1.5.

The setting. Let $M=\Gamma \backslash \mathbb{H}^{3}$ be a rigid acylindrical manifold, with limit set $\Lambda \subset S^{2}$. Note that $\Lambda$ is not contained in a circle, so $\Gamma$ is Zariski dense in $G$.

We will describe 5 possible behaviors for a geodesic plane in $M$, in terms of the orbit closure of a circle, $\overline{\Gamma C} \subset \mathcal{C}$.

Topology of $\boldsymbol{C} \cap \boldsymbol{\Lambda}$. We begin with two observations.

Proposition 7.1 Let $E=C \cap \Lambda$ be the intersection of a circle $C$ with the Sierpiński curve $\Lambda \subset S^{2}$. Then either $E$ is a Cantor set, $E=C$, or $|E| \leq 1$.

Proof. First suppose $E$ has an isolated point $p$. That is, suppose $p=I \cap E$ where $I \subset C$ is an open interval. Since the closures of the components $\left(B_{i}\right)$ of $\Omega$ are disjoint, $I-\{p\}$ must be contained in a single component $B_{i}$. Therefore $C$ is tangent to $\partial B_{i}$ at $p$, and $|E|=1$.

Now suppose $|E|>1$. Then $E$ is perfect, by the preceding argument. If $E \neq C$, then it is also totally disconnected by Theorem 6.1 , and hence $E$ is a Cantor set.

Proposition 7.2 If $|C \cap \Lambda|=0$, then $\Gamma C$ is discrete. If $|C \cap \Lambda|=1$, then $\overline{\Gamma C}=\Gamma \cdot \mathcal{H}(B)$ for some component $B$ of $\Omega$.

Proof. The first case follows from the fact that $\Gamma$ acts properly discontinuously on $\Omega=S^{2}-\Lambda$. In the second case, $C$ is a horocycle for some component $B$ of $\Omega$. Then $\overline{\Gamma C} \neq \mathcal{C}_{\Lambda}$, and we have $D=\partial B \in \overline{\Gamma C}$ by Theorem 3.1, so Theorem 5.2 finishes the proof. 
Exotic circles. We say $C$ is an exotic circle if there is no closed $\Gamma$-orbit in $\overline{\Gamma C}$. (We only regard $C$ as 'exotic' in the rigid acylindrical setting, where there are many closed $\Gamma$-orbits in $\mathcal{C}$.)

The next two sections will be devoted to the proof of the following crucial, technical result.

Theorem 7.3 If $M=\Gamma \backslash \mathbb{H}^{3}$ is a rigid acylindrical manifold, then $\Gamma$ has no exotic circles.

Geometrically, this means the every geodesic plane in $M$ contains a properly immersed geodesic plane in its closure.

Assuming Theorem 7.3, we can now complete the proof the classification theorem for geodesic planes in rigid acylindrical manifolds, using the language of circles.

Proof of Theorem 1.5. Let $M=\Gamma \backslash \mathbb{H}^{3}$ be a rigid acylindrical manifold with limit set $\Lambda$, let $C$ be a circle on $S^{2}$, and let $E=C \cap \Lambda$.

By Proposition 7.1, either $E=C, E$ is a Cantor set or $|E| \leq 1$.

If $|E| \leq 1$, then the behavior of $\overline{\Gamma C}$ is described by Proposition 7.2 above, yielding cases (4) and (5) of Theorem 1.5.

Now assume $|E|>1$ and $\Gamma C$ is discrete. Then $|E|=\infty, E$ coincides with the limit set of $\Gamma^{C}$, and $\Gamma^{C}$ is conjugate to a finitely-generated subgroup of $\mathrm{PGL}_{2}(\mathbb{R})$, by Theorem 6.2. In particular, $\Gamma^{C}$ is nonelementary. Since $\Gamma$ has no parabolics, $\Gamma^{C}$ is convex cocompact, so we have case (1) or (2) of Theorem 1.5.

Finally assume $|E|>1$ and $\Gamma C$ is not closed. Since $\Gamma$ has no exotic circles, there is a closed orbit $\Gamma D \subset \overline{\Gamma C}$. Since $C$ meets $\Lambda$, so does $D$, and hence the limit set of $\Gamma^{D}$ is either a Cantor set or a circle. In either case, $\Gamma^{D}$ is nonelementary, so $\overline{\Gamma C}=\mathcal{C}_{\Lambda}$ by the combination of Theorems 5.1 and 5.2. We cannot have $C \subset \Lambda$, since there are circles in $\overline{\Gamma C}$ that are not contained in $\Lambda$. Hence $E$ is a Cantor set, and we have case (3) of Theorem 1.5.

Remarks. Case (1), where we have $C \subset \Lambda$, can also be treated by doubling core $(M)$ across its boundary and then applying Shah's theorem to the resulting closed manifold.

\section{Unipotent blowup}

In this section we give a self-contained proof of two results concerning the Lie group $G$ which will play a key role in the proof that a rigid acylindrical manifolds has no exotic circles $(\S 9)$. 
Thick sets. Let us say $T \subset \mathbb{R}$ is $K$-thick if

$$
[1, K] \cdot|T|=[0, \infty) .
$$

In other words, given $x \geq 0$ there exists a $t \in T$ with $|t| \in[x, K x]$. We say $T \subset U$ is $K$-thick if its image under an isomorphism $U \cong \mathbb{R}$ is thick.

In this section we will demonstrate two similar results. Recall that $H=$ $\mathrm{PGL}_{2}(\mathbb{R})$, that $U=\left\{\left(\begin{array}{ll}1 & x \\ 0 & 1\end{array}\right): x \in \mathbb{R}\right\}$, and that $V=\left\{\left(\begin{array}{cc}1 & i y \\ 0 & 1\end{array}\right): y \in \mathbb{R}\right\}$. Note that $A N$ (or $V H=A N H$ ) appears in these statements because it is the normalizer of $U$; cf. [Mg1, Lemma 5].

Theorem 8.1 Suppose $g_{n} \rightarrow$ id in $G-V H$. Then there exist $u_{n} \in U$ and $h_{n} \in H$ such that after passing to a subsequence, we have

$$
u_{n} g_{n} h_{n} \rightarrow g \in V-\{i d\}
$$

Moreover, given a sequence of $K$-thick sets $T_{n} \subset U$ and a neighborhood $G_{0}$ of the identity in $G$, we can arrange that $u_{n} \in T_{n}$ and $g \in G_{0}$.

Theorem 8.2 Let $g_{n} \rightarrow$ id in $G-A N$. Then there exist $u_{n}, u_{n}^{\prime} \rightarrow \infty$ in $U$ such that after passing to a subsequence, we have

$$
u_{n} g_{n} u_{n}^{\prime} \rightarrow g \in A V-\{\mathrm{id}\} .
$$

Moreover, given a sequence of $K$-thick sets $T_{n} \subset U$ and a neighborhood $G_{0}$ of the identity in $G$, we can arrange that $u_{n} \in T_{n}$ and $g \in G_{0}$.

In these results, we can think of the unipotent elements $u_{n}$ and $u_{n}^{\prime}$ (or $u_{n}$ and $\left.h_{n}\right)$ as independent changes of coordinates, which blow up or rescale the action of $g_{n}$ to obtain a desirable limit.

Polynomials and thickness. For the proofs we will use the polynomial behavior of unipotent dynamics, together with thickness. The usefulness of these two properties together comes from the following elementary principle.

Let $p_{i}(x) \in \mathbb{R}[x], i=1, \ldots, n$ be a finite sequence of polynomials of total degree $d=\sum \operatorname{deg} p_{i}(x)$, and let $T \subset \mathbb{R}$ be a $K$-thick set. Then there is a constant $k>0$, depending only on $K$ and $d$, such that for any symmetric interval $I=[-a, a]$ we have

$$
\max _{x \in T \cap I} \max _{i}\left|p_{i}(x)\right| \geq k \max _{x \in I} \max _{i}\left|p_{i}(x)\right| .
$$

(Idea of the proof: choose $x \in T \cap I$ such that $x$ is as far as possible from the zeros of the polynomials $p_{i}(x)$.) 
Proof of Theorem 8.1. We will prove the strong form of the result, with respect to a sequence of $K$-thick sets $T_{n}$ and a neighborhood of the origin $G_{0} \subset G$.

It is convenient to pass to the Lie algebra of $H=\mathrm{PGL}_{2}(\mathbb{R})$, given by

$$
\operatorname{Lie}(H)=\left\{\ell=\left(\begin{array}{cc}
a & b \\
c & -a
\end{array}\right): a, b, c \in \mathbb{R}\right\},
$$

with the norm given by

$$
\|\ell\|=\max (|a|,|b|,|c|) .
$$

Note that the Lie algebra of $G$ satisfies

$$
\operatorname{Lie}(G)=\operatorname{Lie}(H) \oplus i \operatorname{Lie}(H)
$$

as a vector space. Thus any element $g \in G$ close to the identity can be factored as

$$
g=\exp (i \ell) h
$$

with $h \in H$ and $\ell \in \operatorname{Lie}(H)$ both small. Choose $\epsilon>0$ such that $\|\ell\|<\epsilon \Longrightarrow$ $\exp (i \ell) \in G_{0}$.

Now suppose $g_{n} \rightarrow$ id in $G-V H$. Then for all $n \gg 0$ we can choose $h_{n} \in H$ and $\ell_{n} \in \operatorname{Lie}(H)$ such that

$$
g_{n} h_{n}=\exp \left(i \ell_{n}\right),
$$

with $h_{n} \rightarrow$ id and

$$
\ell_{n}=\left(\begin{array}{cc}
a_{n} & b_{n} \\
c_{n} & -a_{n}
\end{array}\right) \rightarrow 0 .
$$

By dropping an initial segment of the sequence $\ell_{n}$, we may assume $\left\|\ell_{n}\right\|<\epsilon$ for all $n$. Since $g_{n} \notin V H$, we have:

$$
\left(c_{n} \neq 0\right) \text { or }\left(c_{n}=0 \text { and } a_{n} \neq 0\right) .
$$

Now consider a sequence of unipotent transformations of the form

$$
u_{n}=\left(\begin{array}{cc}
1 & t_{n} \\
0 & 1
\end{array}\right) \in U
$$

We then have

$$
u_{n} \ell_{n} u_{n}^{-1}=\left(\begin{array}{cc}
a_{n}+c_{n} t_{n} & b_{n}-2 a_{n} t_{n}+c_{n} t_{n}^{2} \\
c_{n} & -a_{n}-c_{n} t_{n}
\end{array}\right) .
$$


The norm of the matrix on the right is large when $\left|t_{n}\right|$ is large, by condition (8.3). Since its entries are polynomials in $t_{n}$, and we have $\left\|\ell_{n}\right\|<\epsilon$, the thick set principle expressed in (8.1) implies we can choose $t_{n} \in T_{n}$ such that

$$
k \epsilon \leq\left\|u_{n} \ell_{n} u_{n}^{-1}\right\| \leq \epsilon,
$$

where $k>0$ depends only on $K$.

Since $\ell_{n} \rightarrow 0$, we have $\left|t_{n}\right| \rightarrow \infty$. Pass to a subsequence such that

$$
u_{n} \ell_{n} u_{n}^{-1} \rightarrow \ell \in \operatorname{Lie}(H) .
$$

By construction, we have $k \epsilon \leq\|\ell\| \leq \epsilon$. Since $c_{n} \rightarrow 0, \ell$ is upper triangular. Moreover, $\operatorname{Tr}\left(\ell_{n}^{2}\right) \rightarrow 0$, so $\operatorname{Tr}\left(\ell^{2}\right)=0$, and hence $\ell \in \operatorname{Lie}(U)$. This implies that $g=\exp (i \ell) \in V$. By the upper and lower bounds on $\|\ell\|$, we have $g \in G_{0}-\{$ id $\}$. Since

$$
u_{n} g_{n} h_{n} u_{n}^{-1}=u_{n} \exp \left(i \ell_{n}\right) u_{n}^{-1}=\exp \left(i u_{n} \ell_{n} u_{n}^{-1}\right) \rightarrow \exp (i \ell)=g,
$$

and $h_{n} u_{n}^{-1} \in H$, the proof is complete.

Proof of Theorem 8.2. For this argument we will work in $G$ instead of its Lie algebra. To measure how close an element $g=\left(\begin{array}{ll}a & b \\ c & d\end{array}\right) \in G$ is to the identity, we define

$$
\|g\|=\max \{|a-1|,|b|,|c|,|d-1|\} .
$$

Choose $0<\epsilon<1 / 100$ such that $\|g\|<\epsilon \Longrightarrow\|g\| \in G_{0}$.

Now suppose $g_{n} \rightarrow$ id, where

$$
g_{n}=\left(\begin{array}{ll}
a_{n} & b_{n} \\
c_{n} & d_{n}
\end{array}\right) \in G-A N
$$

After dropping an initial segment of the sequence, we can assume that $\left\|g_{n}\right\|<\epsilon$ for all $n$. Consider a pair of sequences in $U$, given by

$$
u_{n}=\left(\begin{array}{cc}
1 & t_{n} \\
0 & 1
\end{array}\right) \quad \text { and } \quad u_{n}^{\prime}=\left(\begin{array}{cc}
1 & s_{n} \\
0 & 1
\end{array}\right)
$$

Writing $g_{n}^{\prime}=u_{n} g_{n} u_{n}^{\prime}$, we then have

$$
g_{n}^{\prime}=\left(\begin{array}{cc}
a_{n}^{\prime} & b_{n}^{\prime} \\
c_{n}^{\prime} & d_{n}^{\prime}
\end{array}\right)=\left(\begin{array}{cc}
a_{n}+c_{n} t_{n} & b_{n}+d_{n} t_{n}+s_{n}\left(a_{n}+c_{n} t_{n}\right) \\
c_{n} & d_{n}+c_{n} s_{n}
\end{array}\right)
$$


Our goal is to choose $s_{n}$ and $t_{n}$ so that $g_{n}^{\prime}$ converges to a nontrivial matrix of the form

$$
g=\left(\begin{array}{cc}
x & i y \\
0 & x^{-1}
\end{array}\right) \quad \text { with } x, y \in \mathbb{R} .
$$

To this end, we first impose on $t_{n}$ the condition that

$$
\left|c_{n} t_{n}\right|<3 \epsilon
$$

to insure that

$$
1 / 2<\operatorname{Re}\left(a_{n}+c_{n} t_{n}\right)<2 .
$$

Next, we choose $s_{n}$ such that $\operatorname{Re}\left(b_{n}^{\prime}\right)=0$. This gives

$$
s_{n}=-\frac{\operatorname{Re}\left(b_{n}+d_{n} t_{n}\right)}{\operatorname{Re}\left(a_{n}+c_{n} t_{n}\right)} .
$$

Once we have defined $s_{n}$ by (8.8), the entries in $g_{n}^{\prime}$ become rational functions of $t_{n}$, with controlled denominators by (8.7). Thus the entries are comparable to (linear and quadratic) polynomials in $t_{n}$. By the thick set principle expressed in (8.1), we can then choose $t_{n} \in T_{n}$ such that

$$
k \epsilon \leq\left\|g_{n}^{\prime}\right\| \leq \epsilon
$$

where $k>0$ depends only on $K$. It is here we use the fact that $g_{n} \notin A N$ : this insures that either $c_{n} \neq 0$, or $c_{n}=0$ and $\operatorname{Im}\left(a_{n}\right) \neq 0$; in either case, $\left\|g_{n}^{\prime}\right\|$ becomes large if we take $t_{n}$ large, so it eventually exceeds $\epsilon$. Note that $\left\|g_{n}^{\prime}\right\| \leq \epsilon$ implies the condition (8.6) we have assumed above, so we stay in the range with controlled denominators.

Now pass to a subsequence such that $g_{n}^{\prime} \rightarrow g \in G$. By equation (8.9) we have $g \in G_{0}-\{\mathrm{id}\}$. It remains only to verify that $g \in A V$. Clearly $c_{n}=c_{n}^{\prime}$ converges to 0 , and $\operatorname{Re}\left(b_{n}^{\prime}\right)=0$, so the only danger is that the diagonal entries of $g$ might not be real. But in this case $\left|\operatorname{Im}\left(a_{n}^{\prime}\right)\right|=t_{n}\left|\operatorname{Im}\left(c_{n}\right)\right|+o(1)$ would be bounded below for all $n \gg 0$. By (8.5) and the fact that $s_{n} \asymp-t_{n}$, we would then have

$$
\left|\operatorname{Im}\left(b_{n}^{\prime}\right)\right| \asymp t_{n}^{2}\left|\operatorname{Im}\left(c_{n}\right)\right| \rightarrow \infty,
$$

contrary to our choice of $t_{n}$. 


\section{No exotic circles}

In this section we prove that a rigid acylindrical manifold has no exotic circles (Theorem 7.3).

Throughout this section, $M=\Gamma \backslash \mathbb{H}^{3}$ denotes a rigid acylindrical manifold. The stated results hold for all such $M$.

From circles to flows. It is useful to shift perspective from the action of the discrete group $\Gamma$ on the space of circles, $\mathcal{C}=G / H$, to the action of the continuous group $H$ on the frame bundle, FM= $\mathrm{F} \backslash G$.

Let us say an orbit $x H \subset \mathrm{FM}$ is chaotic if $x H \neq \overline{x H}$ and $x H$ meets the renormalized frame bundle $\operatorname{RF} M$. We say $L \subset G$ is a 1-parameter semigroup if $L=\{\exp (t \ell): t \geq 0\}$ for some $\ell \in \operatorname{Lie}(G), \ell \neq 0$.

In this language, we will show:

Theorem 9.1 Let $X \subset \mathrm{F} M$ be a closed set which is a union of chaotic $H$-orbits. Then there is a 1-parameter semigroup $L \subset G$ and $a y \in X$ such that $y L \subset X$ and $L \cap H=\{$ id $\}$.

To prepare for the proof, we must first develop some results on recurrence and unipotent minimal sets.

Thickness. We begin by explaining how thick sets enter the discussion.

Lemma 9.2 There is a $K>1$ such that for every $y \in \operatorname{RF} M$,

$$
T(y)=\{u \in U: y u \in \operatorname{RF} M\}
$$

is a $K$-thick subset of $U$.

Proof. Let $\widetilde{y}=\left(e_{1}, e_{2}, e_{3}\right)$ be a lift of $y$ to a frame at a point $p$ in $\mathbb{H}^{3} \cong$ $\mathbb{C} \times \mathbb{R}_{+}$. We can choose coordinates such that $p=(0,1)$, the geodesic through $e_{1}$ joins $z=0$ to $z=\infty$ in $\partial \mathbb{H}^{3}=\widehat{\mathbb{C}}$, and $e_{2}$ is parallel to the real axis. Since $y$ is in $\operatorname{RF} M$, we have $0, \infty \in \Lambda$. The group $U \cong \mathbb{R}$ acts by translation on $\mathbb{R} \subset \widehat{\mathbb{C}} \cong S^{2}$. If $u \in U$ and $u(0)$ is in $\Lambda$, then the geodesic from $u(0)$ to $u(\infty)=\infty$ joins two points of $\Lambda$, so $u \in T(y)$. Thus it suffices to show that $\Lambda \cap \mathbb{R}$ is uniformly thick in $\mathbb{R}$.

Let $\Omega=\bigcup B_{i}=\widehat{\mathbb{C}}-\Lambda$ be the domain of discontinuity of $\Gamma$, expressed as a union of countably many disjoint round balls. Let

$$
\delta=\inf \left\{d\left(\operatorname{hull}\left(B_{i}\right), \operatorname{hull}\left(B_{j}\right)\right): i \neq j\right\},
$$


where $d$ is the hyperbolic metric on $\mathbb{H}^{3}$. It is easy to see that $\delta$ is positive; for example, $2 \delta$ is bounded below by the length of the shortest closed geodesic on the double of core $(M)$ across its boundary.

Let $I=[t, K t] \subset \mathbb{R}$, where $t>0$ and $K>1$. Choose $K$ such that

$$
d(\operatorname{hull}(I), \operatorname{hull}(-I))=\delta / 2 .
$$

Note that $K$ depends only on $\delta$, since $z \mapsto t z$ is a hyperbolic isometry. Now suppose that $-I \cup I$ is disjoint from $\Lambda$. Since we have $0, \infty \in \Lambda, I$ and $-I$ lie in different components of $\Omega$, say $B_{i}$ and $B_{j}$. It follows that

$$
\delta / 2=d(\operatorname{hull}(I), \operatorname{hull}(-I)) \geq d\left(\operatorname{hull}\left(B_{i}\right), \operatorname{hull}\left(B_{j}\right)\right) \geq \delta,
$$

a contradiction. Thus $-I \cup I$ meets $\Lambda$, and hence $\Lambda \cap \mathbb{R}$ is $K$-thick in $\mathbb{R}$.

Note that convexity of the components of $\Omega \subset \mathbb{C}$ was used in the proof, to insure that $I$ and $-I$ lie in different components of $\Omega$ (because $0 \in \Lambda$ ). This feature would not be present for general acylindrical manifolds, where the components of $\Omega$ are only quasidisks.

Minimality. Let $L$ be a closed subgroup of $G$. We say a set $Y \subset \mathrm{F} M$ is (absolutely) $L-$ minimal if $Y \neq \emptyset$ and

$$
\overline{y L}=Y
$$

for all $y \in Y$. The set $Y$ is relatively $L$-minimal if

$$
Y^{*}=Y \cap \operatorname{RF} M
$$

is nonempty, and (9.2) holds for all $y \in Y^{*}$. Using the Axiom of Choice and compactness of $\mathrm{RF} M$, it is easy to show:

Proposition 9.3 Any closed, L-invariant set meeting RFM contains a relatively L-minimal set.

Translation of $\boldsymbol{Y}$ inside of $\boldsymbol{Y}$. Using thickness, we can now establish an important property of the dynamics of $U$.

Theorem 9.4 For any relatively $U$-minimal set $Y \subset \mathrm{F} M$, there exists a 1-parameter semigroup $L \subset A V$ such that $Y L \subset Y$. 
Theorem 9.4 shows that a unipotent flow automatically gives rise to a whole semigroup of new and unexpected dynamical transformations.

To begin the proof, let

$$
Z=\left\{g \in G: Y^{*} g \cap Y \neq \emptyset\right\},
$$

where $Y^{*}$ is defined by (9.3). Then $Z$ is closed, we have $Z U=Z$, and for every $g \in Z$, there exists a thick set $T \subset U$ such that $T g \subset Z$.

Note that if $g \in A N \cap Z$, then $y g \in Y$ for some $y \in Y^{*}$; and since $g U g^{-1}=U$, we have

$$
Y \supset \overline{y g U}=\overline{y U} g=Y g
$$

by minimality.

Lemma 9.5 For any $y \in Y^{*}$ there exists a sequence $g_{n} \rightarrow$ id in $G-U$ such that $y g_{n} \in Y$.

Proof. By Lemma 9.2 and compactness of $Y^{*}$, we can find $u_{n} \rightarrow \infty$ in $U$ such that $y u_{n} \in Y *$ and $y u_{n} \rightarrow z \in Y^{*}$. In other words, we can write $y u_{n}=z g_{n}$ with $g_{n} \rightarrow$ id in $G$.

Suppose $z=y u$ for some $u \in U$. Then $y u_{n}=z u g_{n}$. Since $\Gamma$ contains no parabolic elements, FM contains no periodic $U$-orbits, and hence $g_{n} \notin G-U$ for all $n \gg 0$. Dropping the finitely many indices with $g_{n} \in U$, we have $u g_{n} u^{-1} \rightarrow$ id in $G-U$, and

$$
y\left(u g_{n} u^{-1}\right)=z g_{n} u^{-1}=y u_{n} u^{-1} \in Y,
$$

for all $n$, by $U$-invariance of $Y$.

Finally suppose $z \notin y U$. Since $z \in Y^{*}$, we have $\overline{z U}=Y$, and thus $z u_{n} \rightarrow y$ for some sequence $u_{n} \in U$. That is, we can write $z u_{n}=y g_{n}$ with $g_{n} \rightarrow$ id in $G$. Then $y g_{n} \in Y$, and $g_{n} \notin U$ since $z \notin y U$.

Proof of Theorem 9.4. By Lemma 9.5, there is a sequence $g_{n} \rightarrow$ id in $G-U$ such that $g_{n} \in Z$. We claim that $Z$ also contains a sequence $z_{n} \in A V$ converging to the identity, $z_{n} \neq$ id.

To see this, first suppose that $g_{n} \in A N$ for infinitely many $n$. Since $Z U=Z$, we can then remove the $U$ component of $g_{n}$ and obtain a sequence $z_{n} \rightarrow$ id in $Z$ with $z_{n} \in A V$. We have $z_{n} \neq$ id since $g_{n} \notin U$.

Now suppose $g_{n} \notin A N$ for all $n \gg 0$. Recall that there is a uniform $K$ and a sequence of $K$-thick sets $T_{n} \subset U$ such that $T_{n} g_{n} U \subset Z$. By Theorem 
8.2, for any neighborhood $G_{0}$ of the identity in $G$, we can choose $u_{n} \in T_{n}$ and $u_{n}^{\prime} \in U_{n}$ such that

$$
u_{n} g_{n} u_{n}^{\prime} \rightarrow z \in A V-\{\mathrm{id}\}
$$

with $z \in G_{0}$. Since $Z$ is closed, we have $z \in Z$; and since the neighborhood $G_{0}$ was arbitrary, we can choose $z_{n} \rightarrow$ id in $Z \cap A V, z_{n} \neq$ id.

By (9.4), every element of the semigroup generated by $z_{n}$ translates $Y$ into itself. Passing to a subsequence and taking a Hausdorff limit, we obtain a closed, 1-parameter semigroup with identity $L \subset A V$ such that $Y L \subset Y$.

Proof of Theorem 9.1. With Theorem 9.4 in place, we can now return to the main line of argument.

Let $X \subset \mathrm{F} M$ be a closed set which is a union of chaotic $H$-orbits. The proof of Theorem 9.1 will depend on a sequence of lemmas, which gradually yield more information about $X$.

As a first simplification, recall that a chaotic $H$-orbit always meets RF $M$. Thus any relatively $H$-minimal subset of $X$ is absolutely minimal. By Proposition 9.3, $X$ contains such a subset. Therefore it suffices to prove Theorem 9.1 under the additional assumption that:

$X$ is an absolutely $H$-minimal set subset of $\mathrm{F} M$.

Using the same Proposition, let us choose a closed set $Y \subset \mathrm{F} M$ such that

$Y$ is a relatively $U$-minimal subset of $X$.

For the remainder of the analysis, we will study the interaction between $X$ and $Y$.

Translation of $\boldsymbol{Y}$ inside of $\boldsymbol{X}$. Our first goal is to move $Y$ inside of $X$. To this end, let

$$
S=\left\{g \in G: Y^{*} g \cap X \neq \emptyset\right\} .
$$

Since $Y^{*}$ is compact, $S$ is closed. Note that $S H=S$; moreover, there is a $K>1$ such that for every $g \in S$, there is a $K$-thick set $T \subset U$ such that $T g \subset S$. Indeed, we can simply take $T=T(y)^{-1}$, where $y \in Y^{*} \cap X g^{-1}$ and $T(y)$ is defined by $(9.1)$.

Lemma 9.6 For any $x \in X$, there exist $g_{n} \rightarrow$ id in $G-H$ such that $x g_{n} \in X$. 
Proof. If not, then there is a neighborhood $G_{0}$ the identity in $G$ such that $x G_{0} \cap X=\left(x G_{0} \cap x H\right)$. This implies that $\left(x G_{0} H\right) \cap X=x H$, and hence the single orbit $x H$ is open in $X$. Since $X$ is $H$-minimal, we have $X=x H$, contrary to our assumption that $X$ contains no closed $H$-orbit.

Lemma 9.7 There exists $a v \in V, v \neq \mathrm{id}$, such that $Y v \subset X$.

Proof. By Lemma 9.6, there is a sequence $g_{n} \rightarrow$ id in $G-H$ such that $g_{n} \in S$. We claim that $S$ also contains a nontrivial element $v \in V$.

First suppose $g_{n} \in V H$ for some $n$. Then we can write $g_{n}=v h$ with $v \in V$ and $h \in H$. Since $S=S H$ and $g_{n} \notin H$, we have $v \in S-\{\operatorname{id}\}$.

Now suppose $g_{n} \notin V H$ for all $n$. Recall that there is a uniform $K$ and a sequence of $K$-thick sets $T_{n} \subset U$ such that $T_{n} g_{n} H \subset S$. By Theorem 8.1, we can choose $u_{n} \in T_{n}$ and $h_{n} \in H$ such that $u_{n} g_{n} h_{n} \rightarrow v \in V-\{$ id $\}$. Since $S$ is closed, we have $v \in S$.

So in either case, $S$ contains a nontrivial element $v \in V$. This means we have a $y \in Y^{*}$ such that $y v \in X$. Since $X$ is $U$-invariant, it follows that

$$
\overline{y v U}=\overline{y U v}=Y v \subset X .
$$

Completion of the proof of Theorem 9.1. By Theorem 9.4 and Lemma 9.7, we have a nontrivial $v \in V$ and a 1-parameter semigroup $L \subset A V$ such that $Y L \subset Y$ and $Y v \subset X$. Choose any $y \in Y$. Then we have $y L \subset X$, so if $L \cap H=\{\operatorname{id}\}$ we are done.

Otherwise, we have $L \subset A$. But then $L^{\prime} \cap H=\{\operatorname{id}\}$, where $L^{\prime}=v^{-1} L v$. Then the point $y^{\prime}=y v$ belongs to $Y v \subset X$, and its $L^{\prime}$ orbit satisfies

$$
y^{\prime} L^{\prime}=y L v \subset Y v \subset X,
$$

completing the proof in this case as well.

Proof of Theorem 7.3. Let $M=\Gamma \backslash \mathbb{H}^{3}$ be a rigid acylindrical manifold, and let $C$ be an exotic circle. This means there is no closed $\Gamma$-orbit in $\overline{\Gamma C} \subset \mathcal{C}=G / H$. In particular, $|D \cap \Lambda|=\infty$ for all $D \in \overline{\Gamma C}$, by Propositions 7.1 and 7.2 .

Let $X \subset \mathrm{F} M=\Gamma \backslash G$ be the unique closed, $H$-invariant set such that $\Gamma X=\overline{\Gamma C} H$ in $G$. Since $C$ is exotic, $X$ has no closed $H$-orbits; and since $|D \cap \Lambda| \geq 2$ for all $D \in \overline{\Gamma C}$, every $H$-orbit in $X$ meets $\mathrm{RF} M$. 
Thus, by Theorem 9.1, there is a 1-parameter semigroup $L \cong \mathbb{R}_{+}$in $G$, and a $y \in X$, such that $L \cap H=\{$ id $\}$ and $y L \subset X$. Then the cosets $y L H \subset X$ give a nonconstant, continuous family of circles $D_{t} \in \overline{\Gamma C}, t \in \mathbb{R}_{+}$. Since $\left|D_{t} \cap \Lambda\right|=\infty$ for all $t, D_{t}$ sweeps out an open subset of $\Lambda$, and hence

$$
\overline{\Gamma D_{t}}=\mathcal{C}_{\Lambda}
$$

for some $t \in \mathbb{R}_{+}$, by Corollary 4.2. Consequently $\overline{\Gamma C}=\mathcal{C}_{\Lambda}$ as well.

Remark. The final argument shows that, in fact, there is no $X \subset \mathrm{F} M$ satisfying the conditions of Theorem 9.1.

\section{Thin Cantor sets}

In this section we prove Theorem 1.6. That is, assuming $M=\Gamma \backslash \mathbb{H}^{3}$ is a rigid acylindrical manifold, and $E=C \cap \Lambda$ is a Cantor set, we will show that $\Gamma C$ is discrete if and only if $E$ is a thin subset of $C$.

On the sphere $S^{n}$, the notion of a thin set can be formulated in terms of hyperbolic geometry. Namely, $E$ is thin if and only if there is an $R>0$ such that hull $(E) \subset \mathbb{H}^{n+1}$ contains no hyperbolic ball of radius $R$. The proof is straightforward.

Thus $E \subset C$ is not thin if and only if the convex hull of $E$, regarded as a subset of the hyperbolic plane $\operatorname{hull}(C) \cong \mathbb{H}^{2}$, contains arbitrarily large hyperbolic balls.

Proof of Theorem 1.6. Suppose $\Gamma C$ is discrete. Then, by Theorem 6.2, $X=\Gamma^{C} \backslash \operatorname{hull}(C)$ is a hyperbolic surface with compact convex core, and $E \subset C$ is the limit set of $\Gamma^{C}$. Since $\operatorname{core}(X)$ is compact, its inradius is bounded above, and the same is true of the convex hull of $E$ in hull $(C)$. Thus $E$ is thin.

Now suppose $\Gamma C$ is not closed. In this case $E$ is not thin because the corresponding geodesic plane $P \subset M$ passes nearly parallel to the boundary of the convex core, and hence $P \cap \operatorname{core}(M)$ contains arbitrarily large balls.

More precisely, since $E$ is Cantor set we have $\overline{\Gamma C}=\mathcal{C}_{\Lambda}$ by Theorem 1.5. Let $\Omega=\bigcup B_{i}$ express the domain of discontinuity as a union of disjoint balls. Note that $\operatorname{diam}\left(B_{i}\right) \rightarrow 0$.

Let $B_{1}=B(x, r)$, and consider the open annulus $A(s)=B(x, r+s)-$ $\overline{B(x, r)}$. Given $\epsilon>0$, choose $s$ small enough that $\operatorname{diam}\left(B_{i}\right)<\epsilon$ whenever $B_{i}$ meets $A(s)$. Since $\Lambda$ is connected, $A(s)$ meets $\Lambda$, and hence it contains a circle centered at $x$ that meets $\Lambda$. Since $\overline{\Gamma C}=\mathcal{C}_{\Lambda}$, there is a nearby circle $C^{\prime}=\gamma C \subset A(s)$ that encloses $B_{1}$. Note that each component of 
$C^{\prime}-E^{\prime}=\bigcup B_{i} \cap C^{\prime}$ has diameter less than $\epsilon$, since $B_{i}$ meets $A(s)$ whenever it meets $C^{\prime}$. Since $\operatorname{diam}\left(C^{\prime}\right)>r$, this implies that hull $\left(E^{\prime}\right) \subset \operatorname{hull}\left(C^{\prime}\right)$ contains a large embedded ball (of hyperbolic radius approximately $\log (1 / \epsilon)$.) But $\gamma$ gives an isometry of $\mathbb{H}^{3}$, sending the convex hull of $E$ to the convex hull of $E^{\prime}$, so hull $(E)$ also contains an arbitrarily large ball, and thus $E$ is not thin.

\section{Isolation and finiteness}

In this section we discuss a general isolation result that follows from the methods of $\S 5$. Geometrically, this result says that compact immersed geodesic surfaces $S \subset M$ tend to repel one another, unless such surfaces are dense.

Theorem 11.1 Let $M=\Gamma \backslash \mathbb{H}^{3}$ be a hyperbolic 3-manifold, and let

$$
X=\left\{C \in \mathcal{C}: S=\Gamma^{C} \backslash \operatorname{hull}(C) \text { is compact }\right\} .
$$

If $\bar{X} \neq \mathcal{C}$, then every point of $X$ is isolated.

Proof. Every circle $C \in X$ is contained in the limit set $\Lambda$ of $\Gamma$, so the same is true for circles in $\bar{X}$. Thus to show $\bar{X}=\mathcal{C}$, it suffices to show that $\bar{X} \supset \mathcal{C}_{\Lambda}$, for this implies that $\Lambda=S^{2}$.

Suppose $C \in X$ is not isolated. Then there is a sequence $C_{i} \neq C$ in $X$ that converges to $C$. Since we have $C_{i} \subset \Lambda$ for all $i$, Corollary 3.2 implies that

$$
\bar{X} \supset \overline{\bigcup \Gamma C_{i}} \supset \mathcal{H}(B)
$$

where $B$ is a component of $S^{2}-C$ meeting $\Lambda$. By Corollary 4.2, this implies that $\bar{X} \supset \mathcal{C}_{\Lambda}$, and hence $\bar{X}=\mathcal{C}$.

Corollary 11.2 Let $M=\Gamma \backslash \mathbb{H}^{3}$ be a convex cocompact 3-manifold. Then either:

- $M$ contains only finitely many immersed, compact geodesic surfaces; or

- $M$ is compact, and the union of all such surfaces is dense. More precisely, compact $H$-orbits are dense in $\mathrm{F} M$. 
Proof. Let $Y=\{C \in \mathcal{C}: C \subset \Lambda\}$, and define $X \subset Y$ by equation (11.1). Note that $Y$ is a closed subset of $\mathcal{C}$, and $X$ consists of isolated points by Theorem 11.1. Since $M$ is convex cocompact, there is a compact set $K \subset \mathcal{C}$ such that every $\Gamma$-orbit in $Y$ meets $K$. If $X \cap K$ is closed, then it is finite, and hence $\Gamma \backslash X$ is finite, which is case (1). Otherwise, there is a circle $C \in \bar{X}-X$. Since $C \in Y, C$ corresponds to a bounded geodesic plane $P \subset M$; and since $C \notin X, M$ is compact and $\bar{P}=M$ by Theorem 1.3. More precisely, we have $\overline{\Gamma C}=\mathcal{C}$ by Theorem B.1 below, and hence $\bar{X}=\mathcal{C}$. This is equivalent to case (2) above.

Corollary 11.3 Let $\Lambda \neq S^{2}$ be the limit set of a convex cocompact Kleinian group $\Gamma$. Then there are finitely many circles $C_{1}, \ldots, C_{n}$ such that

$$
I(\Lambda)=\{C \in \mathcal{C}: C \subset \Lambda\}=\bigcup_{1}^{n} \Gamma C_{i} .
$$

Proof. By Theorem 1.3, every circle $C \subset \Lambda$ gives a compact immersed geodesic surface $S \subset M$.

Arithmetic 3-manifolds and open surfaces. By the preceding results, a rigid acylindrical manifold $M$ contains only finitely many compact immersed geodesic surfaces. We conclude by showing that the situation for open surfaces is different. It is convenient to define, for any Kleinian group $\Gamma$,

$$
\mathcal{D}(\Gamma)=\{C \in \mathcal{C}: \Gamma C \text { is discrete in } \mathcal{C}\}
$$

Theorem 11.4 Let $M$ be a covering space of a compact arithmetic 3-manifold, and suppose there is a compact $H$-orbit in FM. Then closed $H$-orbits are dense in $\mathrm{F} M$.

Proof. Let $M_{0}=\Gamma_{0} \backslash \mathbb{H}^{3}$ be a compact arithmetic 3-manifold, and let $M$ be the covering space of $M_{0}$ determined by a subgroup $\Gamma \subset \Gamma_{0}$. Then $M_{0}$ inherits at least one immersed, compact totally geodesic surface from the compact $H$-orbit in $M$. By [Re], the presence of one such surface guarantees that $M_{0}$ contains infinitely many of them. By Corollary 11.2 above, this means that $\mathcal{D}\left(\Gamma_{0}\right)$ is dense in $\mathcal{C}$. Since $\mathcal{D}(\Gamma)$ contains $\mathcal{D}\left(\Gamma_{0}\right)$, and discrete $\Gamma$-orbits in $\mathcal{C}=G / H$ correspond to closed $H$-orbits in $\mathrm{F} M=\Gamma \backslash G$, the latter are dense as well. 
Corollary 11.5 Let $M=\Gamma \backslash \mathbb{H}^{3}$ be a rigid acylindrical 3-manifold that covers a compact arithmetic 3-manifold. Then there are infinitely many open, properly immersed geodesic surfaces passing through the convex core of $M$.

These surfaces correspond to case (2) in Theorem 1.1.

Proof. The components of $\partial \operatorname{core}(M)$ give compact $H$-orbits in $\mathrm{F} M$, so closed $H$-orbits are dense. Equivalently, $\mathcal{D}(\Gamma)$ is dense in $\mathcal{C}$. The circles in $\mathcal{D}(\Gamma)$ that meet $\Lambda$ in a Cantor set provide the desired surfaces in $M$.

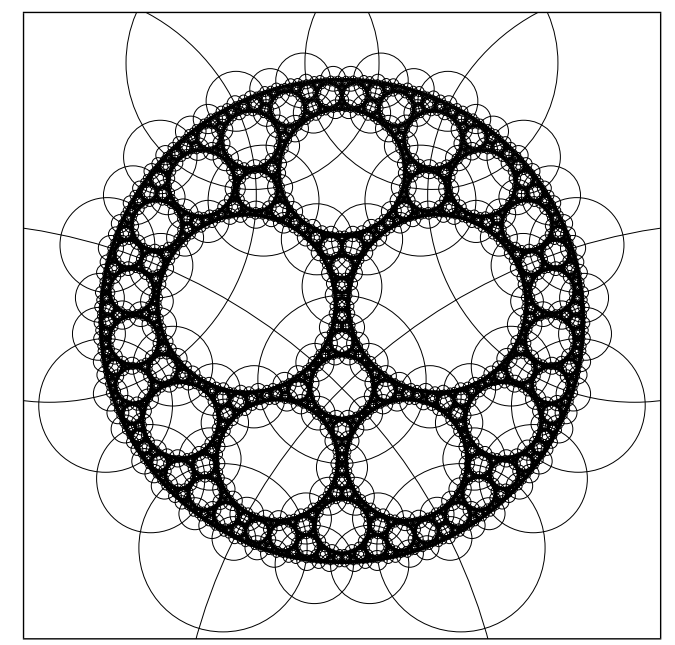

Figure 3. A rigid acylindrical subgroup of an arithmetic group.

Example. Figure 3 illustrates an arithmetic rigid acylindrical manifold with invariant trace field $\mathbb{Q}(\sqrt{-7})$. The Figure shows the orbit of a circle $C$ such that $C \cap \Lambda$ is a Cantor set, and $\Gamma C \subset \mathcal{C}$ is discrete. The circles with this behavior are dense in $\mathcal{C}$ by Theorem 11.4. In this example, $\Gamma$ is a subgroup of one of the compact arithmetic tetrahedral reflection groups, enumerated in $[\mathrm{MR} 2, \S 13]$.

\section{A Appendix: Quasifuchsian groups}

In this section we show that for a quasifuchsian group $\Gamma$, the closure of a geodesic plane in $M=\Gamma \backslash \mathbb{H}^{3}$ passing through the convex core can be rather wild. The case of a Fuchsian group was treated in the Introduction, and we will show the same phenomena persists under a small bending deformation. 
Bending. Let $\Sigma_{0}=\Gamma_{0} \backslash \mathbb{H}^{2}$ be a closed hyperbolic surface of genus $g \geq 2$. We can identify $\Sigma_{0}$ with the convex core of the 3-manifold $M_{0}=\Gamma_{0} \backslash \mathbb{H}^{3}$, which can be written as

$$
M_{0} \cong \Sigma_{0} \times \mathbb{R}
$$

in cylindrical coordinates. Here the first coordinate is given by the nearestpoint projection $\pi: M_{0} \rightarrow \Sigma_{0}$, and the second is given by the signed distance from $\Sigma_{0}$.

Let $\beta \subset \Sigma_{0}$ be a simple closed curve. Then for all angles $\theta$ sufficiently small, there is a quasifuchsian manifold $M_{\theta}=\Gamma_{\theta} \backslash \mathbb{H}^{3}$ and a path isometry

$$
j_{\theta}: \Sigma_{0} \rightarrow \Sigma_{\theta} \subset \partial \operatorname{core}\left(M_{\theta}\right),
$$

such that $\Sigma_{\theta}$ is bent with a dihedral angle of $\theta$ along the image of $\beta$, and otherwise totally geodesic. The manifold $M_{\theta}$ is unique up to isometry. See e.g. [Th1, Ch. 8], [EpM], [KaT], [Mc2] for this construction.

Choose $r$ sufficiently small that $\beta$ has an embedded annular collar neighborhood $B_{r}$ of width $2 r$. Removing this collar, we obtain a surface with boundary

$$
\Sigma_{0}(r)=\Sigma_{0}-B_{r} \subset \Sigma_{0},
$$

and a closed submanifold

$$
M_{0}(r)=\Sigma_{0}(r) \times \mathbb{R} \subset M_{0} .
$$

There is a unique extension of $j_{\theta} \mid \Sigma_{0}(r)$ to an orientation-preserving isometric immersion

$$
J_{\theta}: M_{0}(r) \rightarrow M_{\theta}
$$

which sends geodesics normal to $\Sigma_{0}(r)$ to geodesics normal to $\Sigma_{\theta}(r)=$ $j_{\theta}\left(\Sigma_{0}(r)\right)$.

Proposition A.1 Assume $M_{\theta}$ is a quasifuchsian manifold, and $\sin (|\theta| / 2)<$ $\tanh (r)$. Then the map $J_{\theta}: M_{0}(r) \rightarrow M_{\theta}$ is a proper, isometric embedding.

Sketch of the proof. Qualitatively, the statement above is nearly immediate from the divergence of geodesics normal to $\Sigma_{0}$ (see Figure 4 ). The extreme case $\sin (\theta / 2)=\tanh (r)$ corresponds to a hyperbolic right triangle with base of length $r$ and one ideal vertex; its internal angles are $(0, \pi, \pi-\theta / 2)$. 

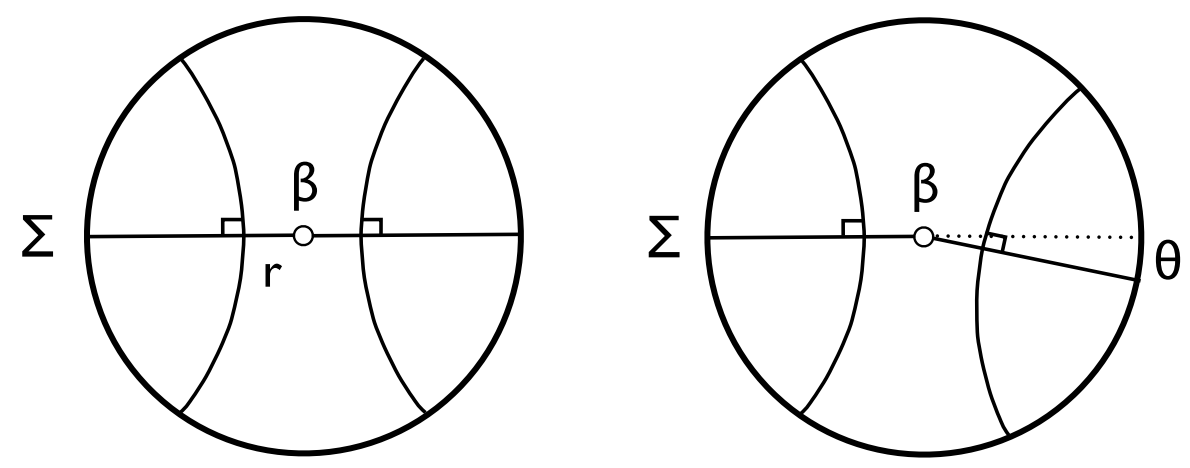

Figure 4. Geodesics normal to $\Sigma_{0}$ and at least distance $r$ from the bending locus remain disjoint under small bendings. (Diagram is in the universal cover.)

Planes in quasifuchsian manifolds. Now let $\gamma \subset \Sigma_{0}$ be an immersed geodesic such that $\bar{\gamma}$ is disjoint from $\beta$. Then we can also choose $r$ sufficiently small that $\bar{\gamma}$ lies in $\Sigma_{0}(r)$. As discussed in $\S 1$, the locus $P=\gamma \times \mathbb{R} \subset M_{0}$ is an immersed geodesic plane with

$$
\bar{P}=\bar{\gamma} \times \mathbb{R} \subset M_{0} .
$$

But in fact we have $\bar{P} \subset M_{0}(r)$. Thus $P_{\theta}=J_{\theta}(P)$ is an immersed geodesic plane in $M_{\theta}$. By Proposition A.1, for all $\theta$ sufficiently small, $J_{\theta}$ maps a neighborhood of $\bar{P}$ isometrically to a neighborhood of $\bar{P}_{\theta}$. This shows:

Corollary A.2 For all $\theta$ sufficiently small, the quasifuchsian manifold $M_{\theta}$ contains an immersed geodesic plane $P_{\theta}$, passing through its convex core, such

$$
\bar{P}_{\theta} \cong \bar{P}=\bar{\gamma} \times \mathbb{R} \subset M_{0} .
$$

As noted in $\S 1$, the closure of a geodesic can be rather wild, and the same is true for geodesics with $\bar{\gamma}$ disjoint from $\beta$. In particular, the closure of $P_{\theta}$ need not be a submanifold of $M_{\theta}$, so we have no analogue of Corollary 1.2 .

\section{B Appendix: Bounded geodesic planes}

In this section we record a rigidity theorem for bounded geodesic planes in an arbitrary hyperbolic 3-manifold. Shah's theorem on geodesic planes in a compact hyperbolic 3-manifold [Sh] follows as a special case. 
Theorem B.1 Let $P \subset M$ be a bounded geodesic plane in an arbitrary hyperbolic 3-manifold $M=\Gamma \backslash \mathbb{H}^{3}$. Then either:

- $P$ is a compact, immersed hyperbolic surface; or

- $M$ is compact, and $\bar{P}=M$. More precisely, frames tangent to $P$ are dense in $\mathrm{F} M$.

Proof. The proof will follow the same lines as the proof in $\S 9$ above.

Let $\Lambda \subset S^{2}$ be the limit set of $\Gamma$, and let $C \subset S^{2}$ be a circle corresponding to a lift of $P$ to the universal cover of $M$. To prove the theorem, we must show that $\Gamma C \subset \mathcal{C}$ is either closed or dense. Note we have $C \subset \Lambda$, because $P$ is bounded in $M$. Thus every circle $D$ in $\overline{\Gamma C}$ is also contained in $\Lambda$.

Assume $\Gamma C$ is not closed. We will show that $\overline{\Gamma C}=\mathcal{C}_{\Lambda}$.

Since $\Gamma C$ is not closed, $\Gamma$ is Zariski dense in $G$, and there is limit set on both sides of $C$. (Otherwise $C$ would be the boundary of a component of $\Omega=S^{2}-\Lambda$, and hence $\Gamma C$ would be discrete.)

If $\Gamma D$ is closed for some $D \in \overline{\Gamma C}$, then $\overline{\Gamma C}=\mathcal{C}_{\Lambda}$ by Theorems 5.1 and 6.2. So we may assume that $C$ is an exotic circle, i.e. $\overline{\Gamma D} \neq \Gamma D$ for all $D \in \overline{\Gamma C}$. Then the corresponding compact, $H$-invariant set $X \subset \mathrm{F} M$ contains no closed $H$-orbit.

Since $P$ is compact, and $C$ is contained in $\Lambda, X$ is a compact subset of the renormalized frame bundle RF $M$.

The argument of $\S 9$ now goes through verbatim, to show that $\overline{\Gamma C}=\mathcal{C}_{\Lambda}$. Indeed, the argument is somewhat simpler: since $X \subset \operatorname{RF} M$, we have $Y^{*}=$ $Y$, and hence there is no need to consider thick sets (i.e. we can take $T_{n}=U$ when applying Theorems 8.1 and 8.2).

To complete the proof, note that every circle in $\overline{\Gamma C}$ is contained in the limit set. Thus $\overline{\Gamma C}=\mathcal{C}_{\Lambda}$ implies $\Lambda=S^{2}$ and $\overline{\Gamma C}=\mathcal{C}$. Consequently frames tangent to $P$ are dense in $\mathrm{F} M$; and since $P$ is bounded, $M$ is compact.

Using Theorem 11.1, we obtain the following analogue of Corollary 11.2:

Corollary B.2 Let $K \subset M$ be a compact subset of a hyperbolic 3-manifold. Then either:

- The set of compact geodesic surfaces in $M$ contained in $K$ is finite; or

- Compact geodesic surfaces are dense in $M$, and $M$ is compact.

Remarks. One could also deduce these results from Ratner's works [Rn1] and [Rn2], using [DM, Thm. 1] or modifying [Rn2, Thm 2.1] to handle the case where $\Gamma$ is not a lattice. 


\section{References}

[Da] F. Dal'bo. Topologie du feuilletage fortement stable. Ann. Inst. Fourier 50(2000), 981-993.

$[\mathrm{DM}]$ S. G. Dani and G. A. Margulis. Limit distributions of orbits of unipotent flows and values of quadratic forms. In I. M. Gelfand Seminar, volume 16 of Adv. Soviet Math., pages 91-137. Amer. Math. Soc., 1993.

[Eb] P. Eberlein. Geodesic flows on negatively curved manifolds. I. Ann. of Math. 95(1972), 492-510.

[EpM] D. B. A. Epstein and A. Marden. Convex hulls in hyperbolic space, a theorem of Sullivan, and measured pleated surfaces. In Analytical and Geometric Aspects of Hyperbolic Space, pages 113-254. Cambridge University Press, 1987.

[Ein] M. L. Einsiedler et al, editor. Homogeneous flows, moduli spaces and arithmetic, volume 10 of Clay Mathematics Proceedings. American Mathematical Society, 2010.

[Fer] D. Ferte. Flot horosphérique des repères sur les variétés hyperboliques de dimension 3 et spectre des groupes kleiniens. Bull. Braz. Math. Soc. 33(2002), 99-123.

[Hed] G. A. Hedlund. Fuchsian groups and transitive horocycles. Duke Math. J 2(1936), 530-542.

[KaT $]$ Y. Kamishima and Ser P. Tan. Deformation spaces on geometric structures. In Y. Matsumoto and S. Morita, editors, Aspects of Low Dimensional Manifolds, pages 263-300. Published for Math. Soc. of Japan by Kinokuniya Co., 1992.

[MR1] C. Maclachlan and A. W. Reid. Commensurability classes of arithmetic Kleinian groups and their Fuchsian subgroups. Math. Proc. Camb. Phil. Soc. 102(1987), 251-257.

[MR2] C. Maclachlan and A. W. Reid. The Arithmetic of Hyperbolic 3Manifolds. Springer-Verlag, 2003.

[Mg1] G. A. Margulis. Indefinite quadratic forms and unipotent flows on homogeneous spaces. In Dynamical systems and ergodic theory (Warsaw, 1986), volume 23. Banach Center Publ., 1989. 
[Mg2] G. A. Margulis. Dynamical and ergodic properties of subgroup actions on homogeneous spaces with applications to number theory. In Proceedings of the International Congress of Mathematicians (Kyoto, 1990), pages 193-215. Springer-Verlag, 1991.

[MT] K. Matsuzaki and M. Taniguchi. Hyperbolic Manifolds and Kleinian Groups. Oxford University Press, 1998.

[Mc1] C. McMullen. Iteration on Teichmüller space. Invent. math. 99(1990), 425-454.

[Mc2] C. McMullen. Complex earthquakes and Teichmüller theory. J. Amer. Math. Soc. 11(1998), 283-320.

[Mc3] C. McMullen. Teichmüller geodesics of infinite complexity. Acta Math. 191(2003), 191-223.

[MMO] C. McMullen, A. Mohammadi, and H. Oh. Horocycles in hyperbolic 3-manifolds. Preprint, 2015.

[Pa] T. L. Payne. Closures of totally geodesic immersions into locally symmetric spaces of noncompact type. Proc. Amer. Math. Soc. $127(1999)$, 829-833.

[PR $\quad$ G. Prasad and A. S. Rapinchuk. Existence of irreducible $\mathbb{R}$-regular elements in Zariski-dense subgroups. Math. Res. Lett. 10(2003), $21-32$.

[Rc] J. G. Ratcliffe. Foundations of Hyperbolic Manifolds. SpringerVerlag, 1994.

[Rn1] M. Ratner. On Raghunathan's measure conjecture. Annals of Math. 134(1991), 545-607.

[Rn2] M. Ratner. Raghunathan's topological conjecture and distributions of unipotent flows. Duke Math. J. 63(1991), 235-280.

[Rn3] M. Ratner. Interactions between ergodic theory, Lie groups and number theory. In Proceedings of the International Congress of Mathematicians (Zürich, 1994), pages 156-182. Birkhaüser, Basel, 1995.

[Re] A. W. Reid. Totally geodesic surfaces in hyperbolic 3-manifolds. Proc. Edinburgh Math. Soc. 34(1991), 77-88. 
[Sh] N. A. Shah. Closures of totally geodesic immersions in manifolds of constant negative curvature. In Group Theory from a Geometrical Viewpoint (Trieste, 1990), pages 718-732. World Scientific, 1991.

[Sul] D. Sullivan. Related aspects of positivity: $\lambda$-potential theory on manifolds, lowest eigenstates, Hausdorff geometry, renormalized Markoff processes .... In Aspects of mathematics and its applications, volume 34, pages 747-779. North-Holland, 1986.

[Th1] W. P. Thurston. Geometry and Topology of Three-Manifolds. Lecture Notes, Princeton University, 1979.

[Th2] W. P. Thurston. Hyperbolic structures on 3-manifolds I: Deformations of acylindrical manifolds. Annals of Math. 124(1986), 203246.

[Why] G. T. Whyburn. Topological characterization of the Sierpiński curve. Fund. Math. 45(1958), 320-324.

[Win] D. Winter. Mixing of frame flow for rank one locally symmetric spaces and measure classification. Israel J. Math. 210(2015), 467507.

Mathematics Department, Harvard University, 1 Oxford St, CAmbridge, MA 02138-2901

Mathematics Department, University of Texas at Austin, 1 University Station C1200, Austin, TX 78712

Mathematics Department, Yale University, 10 Hillhouse Avenue, New Haven, CT 06511, and Korea Institute for Advanced Study, Seoul, Korea 